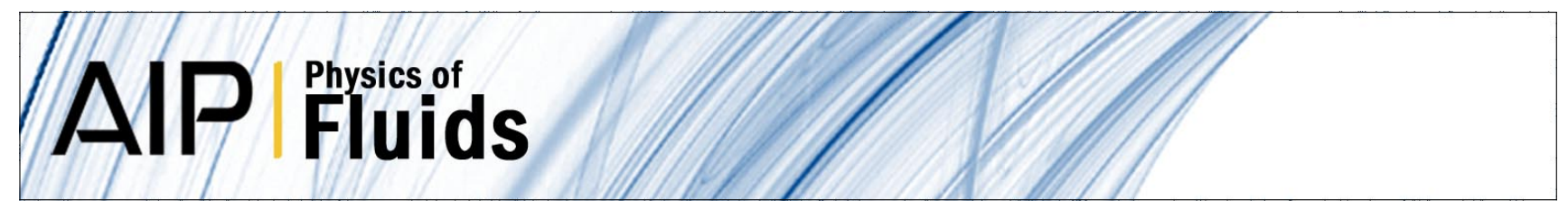

\title{
The planar singular solutions of Stokes and Laplace equations and their application to transport processes near porous surfaces
}

\author{
A. S. Sangani and S. Behl
}

Citation: Phys. Fluids A 1, 21 (1989); doi: 10.1063/1.857544

View online: http://dx.doi.org/10.1063/1.857544

View Table of Contents: http://pof.aip.org/resource/1/PFADEB/v1/i1

Published by the American Institute of Physics.

\section{Related Articles}

Clouds of particles in a periodic shear flow

Phys. Fluids 24, 021703 (2012)

The dynamics of a vesicle in a wall-bound shear flow

Phys. Fluids 23, 121901 (2011)

A study of thermal counterflow using particle tracking velocimetry

Phys. Fluids 23, 107102 (2011)

Particle accumulation on periodic orbits by repeated free surface collisions

Phys. Fluids 23, 072106 (2011)

Drag force of a particle moving axisymmetrically in open or closed cavities

J. Chem. Phys. 135, 014904 (2011)

\section{Additional information on Phys. Fluids A}

Journal Homepage: http://pof.aip.org/

Journal Information: http://pof.aip.org/about/about_the_journal

Top downloads: http://pof.aip.org/features/most_downloaded

Information for Authors: http://pof.aip.org/authors

\section{ADVERTISEMENT}

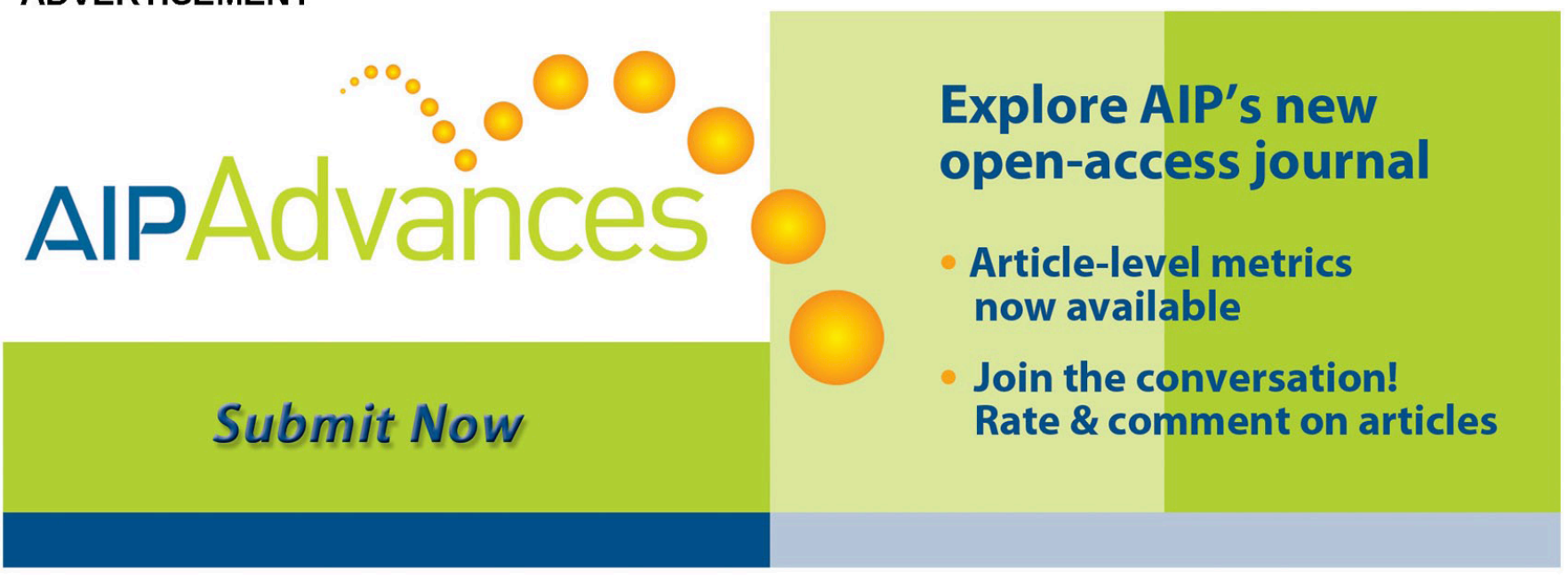




\title{
The planar singular solutions of Stokes and Laplace equations and their application to transport processes near porous surfaces
}

\author{
A. S. Sangani and S. Behl \\ Department of Chemical Engineering and Materials Science, Syracuse University, Syracuse, New York \\ 13244
}

(Received 27 July 1988; accepted 12 September 1988)

\begin{abstract}
The planar singular solutions of Stokes and Laplace equations are derived and applied to a number of transport problems associated with porous surfaces. The velocity, pressure, concentration, and temperature slip coefficients are determined exactly for the semi-infinite periodic arrays of spheres and these are compared with the predictions of two approximate continuum theories formulated by Brinkman [Appl. Sci. Res. Sect. A 1, 27 (1947)] and Chang and Acrivos [J. Appl. Phys. 59, 375 (1986)] to assess the utility of such theories in accurately predicting various overall properties related to the porous surfaces. It is found that in general these theories provide fairly accurate estimates of these properties even when the length scales based on the relevant macroscopic properties such as the permeability are much smaller than the length scales characterizing the microstructure of the porous media.
\end{abstract}

\section{INTRODUCTION}

Considerable progress has been made in recent years in our ability to estimate quantitatively overall macroscopic properties such as conductivity, Darcy permeability, viscosity, and the sedimentation coefficient of suspensions of spherical or cylindrical particles when the suspensions are homogeneous on a macroscale, i.e., a length scale large compared to the dimensions of the particles. A number of numerical techniques have appeared in the literature to solve the many-particle interactions either in a rigorous manner or in an approximate manner (see, for example, Refs. 1-8). Similarly, a number of continuum theories have also been developed for predicting the macroscopic transport properties, which, although somewhat nonrigorous, give estimates of the macroscopic properties that are in reasonable agreement with their actual values determined either experimentally or numerically via the many-particle calculations (see, for example, Refs. 7-10). These continuum theories have been extended recently also to treat the problems in which either the suspension does not appear homogeneous on the macroscale or the length scale on which a transport process such as the heat conduction occurs is comparable to the dimension of the particles. ${ }^{11-13}$ Since the exact numerical or experimental results for this latter category of problems are not widely available in the literature, our present efforts are directed toward developing efficient numerical techniques to solve such problems.

In this paper we present a technique suitable for solving the transport problems in the vicinity of an "interface" of a suspension with a fluid. Note that since the same fluid is also the continuous or the suspending medium in the suspension, the "interface" between the suspension and the fluid is unlike the usual interfaces that imply a clear boundary between two phases. Although both "phases," i.e., the fluid and the suspension, appear homogeneous on suitably defined macroscales, it is clear that for any transport process occurring near the "interface," which typically spreads a few diameters wide into both "phases," the media cannot be treated as ho- mogeneous. In fact, the precise definition of where the "interface" is located is necessary, in general, to make a meaningful comparison between the exact numerical results and the approximate continuum theories. Since the variation of the pressure, velocity, or temperature with the distance from the "interface" is of primary interest, we shall consider here a somewhat idealized situation in which the suspension, which occupies a half-space $x_{1}>0$, consists of a continuous fluid medium and the spherical particles whose centers are located on the planes equidistant from each other and with the centers of the particles on any given plane forming a planar periodic lattice, the period of all the lattices being the same. In other words, we shall consider various transport problems for the semi-infinite periodic suspensions. When the particles are held fixed, the suspension serves as a model for a granular porous medium or a filter cake.

Recently, Larson and Higdon ${ }^{14,15}$ have determined the detailed velocity and pressure distributions for a shear flow past a semi-infinite array of infinitely long parallel cylinders. They considered both the cases of mean flow parallel as well as perpendicular to the axes of the cylinders and solved the Poisson and Stokes equations numerically using a boundaryintegral method. In addition to the detailed calculations of the velocity and pressure distributions, these authors also reported the slip coefficients (defined in Sec. III) for various volume fractions of the cylinders arranged in a semi-infinite array. The approximate theory of Brinkman and the work of other investigators on this problem have been summarized in detail by Larson and Higdon and hence we shall not repeat it here. The necessary points relevant to our calculations will be discussed in Sec. III where this problem is treated in detail.

The numerical technique we have developed is different from the one employed by Larson and Higdon and it makes use of the planar singular solutions of the Laplace and Stokes equations. These singular solutions were first developed by Ishii ${ }^{16}$ who used them to determine the flow in the entrance region of the semi-infinite array, i.e., for the case of a mean 
flow along the $x_{1}$ direction. Using these solutions, Ishii estimated the drag forces on the spherical particles arranged in a semi-infinite array when the volume fractions of the particles are small. This method has been extended here to obtain numerical results for the complete range of volume fractions of the spherical particles. Briefly, the velocity field in the fluid is first expressed in terms of various derivatives of the singular solutions of the governing equations with the singularities located inside each particle. It is assumed that the velocity field around each particle in any given $x_{1}$ plane is identical and hence the strengths of the singularities vary only among the particles located in different $x_{1}$ planes. These strengths are determined by satisfying the appropriate boundary conditions on the surface of the particles. Using this technique, we obtain the solutions of the following four problems: (i) a slip flow or the shear flow past a porous surface; (ii) a normal flow in the entrance region of a porous medium; (iii) a thermal conduction near the boundary of a composite medium; and (iv) a diffusion accompanied by a chemical reaction near a porous catalyst. For each of these problems, a comparison with appropriate continuum theories is also made to assess the utility of these theories in correctly predicting the quantitative behavior of the transport processes near the "interface" of the suspensions and the fluid. In particular, we find that an approximate method recently proposed by Chang and Acrivos ${ }^{9-11}$ provides an excellent estimate of the slip coefficient in problem (i) for the complete range of volume fractions of the spheres but fails to give the correct estimates for problems (ii) and (iii), in which the pressure and temperature slips are determined, in that the approximate theory predicts zero values for these slips whereas the exact calculations give small but nonzero values of these quantities. In view of the rather small numerical values of these slips, however, the failure of the approximate method for these two problems must be regarded as minor. Finally, an approximate theory formulated by Brinkman $^{17}$ gives better estimates in the case of problem (iv), where the comparison is limited to slow to moderately fast reactions. By combining the results of exact calculations with the continuum theory of Brinkman, we also estimate the bulk diffusivities of porous media in the presence of a chemical reaction. Our detailed comparison of the exact results with the approximate theories for these four problems suggests that, in general, the approximate theories provide fairly accurate estimates of the transport coefficients related to the "interfaces" of porous media with the continuous fluid. In particular, the approximate theories give very good estimates even when the ratio of the length scales based on the relevant macroscopic properties, such as the permeability, to the microstructure length scale, is much smaller than unity.

We wish to note that although in a number of problems, such as the convective heat transfer, the finite nature of the "interface" or its curvature must be taken into account for a proper modeling of the transport phenomenon near the "interface," the solutions presented in this paper, which assume identical temperature or pressure fields around each sphere in a given $x_{1}$ plane, will be useful in the analysis of the "inner region" near the "interface."
The outline of the paper is as follows. In Sec. II we shall begin with the definition and description of the planar singular solutions and their derivatives. In Secs. III-VI these singular solutions are employed for determining the solutions of the four problems listed earlier and the numerical results for various interface-related properties thus obtained are compared with the approximate continuum theories. In Sec. VII, we summarize the important conclusions of the study.

\section{THE PLANAR SINGULAR SOLUTIONS OF THE STOKES AND LAPLACE EQUATIONS}

Since the planar periodic singular solutions are employed in constructing the solutions of the problems considered in this paper, we describe them in detail here.

A fundamental singular solution of the Stokes equations of motion satisfies

$$
\begin{aligned}
& \frac{\partial u_{i}}{\partial x_{i}}=0, \\
& \nabla^{2} u_{i}=\frac{\partial P}{\partial x_{i}}+F_{i}^{\alpha} \sum_{T} \delta\left(\mathbf{r}-\mathbf{r}^{\alpha}-\mathbf{r}^{\prime}\right),
\end{aligned}
$$

where $\mathbf{r}^{\alpha}$ represents the position vector of a representative lattice point on the plane $x_{1}=x_{1}^{\alpha}, u_{i}$ and $P$ are the velocity and pressure, $F_{i}^{\alpha}$ is a constant, and $\mathbf{r}^{\prime}$ are the lattice vectors given by

$$
\mathbf{r}^{1}=h\left(l_{2} \mathbf{a}_{2}+l_{3} \mathbf{a}_{3}\right), \quad l_{2}, l_{3}=0, \pm 1, \pm 2, \pm 3, \ldots .
$$

Here $h$ is a length characteristic of the lattice dimension and $\mathbf{a}_{2}$ and $\mathbf{a}_{3}$ are the dimensionless basis vectors of the planar array. Physically, $u_{i}$ and $P$ correspond to the velocity and pressure fields caused by point forces $F_{i}^{a}$ acting on the fluid through the lattice points on the plane $x_{1}=x_{1}^{a}$.

The solution of (1) and (2) subject to the condition that $u_{i}$ and $P$ must vanish at infinity was obtained by Ishii, ${ }^{16}$ who expressed it in terms of two functions which he referred to as $S_{1}$ and $S_{2}$. Since we have employed the symbols $S_{1}$ and $S_{2}$ in the past to denote the singular solutions of the three-dimensional lattice, we shall refer to Ishii's functions by $\Psi_{1}$ and $\Psi_{2}$. Thus

$$
\begin{aligned}
& u_{i}(\mathbf{r})=-F_{j}^{\alpha} v_{i j}\left(\mathbf{r}-\mathbf{r}^{\alpha}\right), \\
& P(\mathbf{r})=-F_{j}^{\alpha} q_{j}\left(\mathbf{r}-\mathbf{r}^{\alpha}\right),
\end{aligned}
$$

with

$$
\begin{aligned}
& 4 \pi v_{i j}=\delta_{i j} \Psi_{1}-\frac{\partial^{2} \Psi_{2}}{\partial x_{i} \partial x_{j}}, \\
& \frac{\partial q_{j}}{\partial x_{i}}=\tau^{-1} \delta_{i j} \delta\left(x_{1}-x_{1}^{\alpha}\right)-\frac{1}{4 \pi} \frac{\partial^{2} \Psi_{1}}{\partial x_{i} \partial x_{j}} .
\end{aligned}
$$

The functions $\Psi_{1}$ and $\Psi_{2}$ are given by

$\Psi_{m}(\mathbf{r})$

$$
\begin{aligned}
= & \frac{1}{\pi \tau}\left(\frac{-1}{4 \pi^{2}}\right)^{m-1} \int_{-\infty}^{\infty} d k \sum_{1}^{\prime} Q^{-2 m} e^{-2 \pi \mathbf{Q} \cdot \mathbf{r},} \\
& m=1,2,
\end{aligned}
$$

with

$$
\begin{aligned}
& \mathbf{Q}=k \mathbf{e}_{1}+l_{2} \mathbf{b}_{2}+l_{3} \mathbf{b}_{3}, \quad \mathbf{I}=\left(l_{2}, l_{3}\right), \\
& \mathbf{Q} \cdot \mathbf{r}=k x_{1}+q_{2} x_{2}+q_{3} x_{3} .
\end{aligned}
$$


Here $e_{1}$ is a unit vector along the $x_{1}$ axis, $b_{2}$ and $b_{3}$ are the basis lattice vectors in the reciprocal lattice, and $q_{2}$ and $q_{3}$ are related to the components of $b_{2}, b_{3}$, and $\mathbf{l}$. The prime above the summation sign in (8) implies that the term with $l_{2}=l_{3}=0$ is excluded from the summation. The area of the unit cell for the planar lattice is denoted by $\tau$.

The functions $\Psi_{1}$ and $\Psi_{2}$ satisfy the following differential equations:

$$
\begin{aligned}
& \nabla^{2} \Psi_{1}(\mathbf{r})=4 \pi\left(\frac{\delta\left(x_{1}\right)}{\tau}-\sum_{T} \delta\left(\mathbf{r}-\mathbf{r}_{1}\right)\right), \\
& \nabla^{2} \Psi_{2}=\Psi_{1} .
\end{aligned}
$$

In view of (11), $\Psi_{1}$ may be regarded as a solution of a thermal conduction problem with sources of thermal energy situated at the lattice points surrounded by sinks of uniform density on the plane $x_{1}=0$. Because of this property of $\Psi_{1}$, it is readily seen that $\Psi_{1}$ represents the periodic singular solution of the Laplace equation. Since we shall need to evaluate various derivatives of $\Psi_{1}$ and $\Psi_{2}$, we next describe three different methods to evaluate the derivatives.

\section{A. The first method}

This method is suitable for $\left|x_{1}\right|$ greater than, say, $h / 2$. Integrating (8) with respect to $k$ we obtain, for $m=1$,

$$
\Psi_{1}(\mathbf{r})=\sum_{1}^{\prime} \frac{1}{\tau q_{1}} e^{-2 \pi i q_{1} \cdot s} e^{-2 \pi q_{1}\left|x_{1}\right|},
$$

where

$$
\begin{aligned}
& \mathbf{q}_{1}=q_{2} \mathbf{e}_{2}+q_{3} \mathbf{e}_{3}, \\
& \mathbf{s}=x_{2} \mathbf{e}_{2}+x_{3} \mathbf{e}_{3} .
\end{aligned}
$$

The above expression for $\Psi_{1}$ can be readily differentiated. Thus, for example,

$$
\begin{aligned}
\left(\frac{\partial}{\partial x_{1}}\right)^{n-m} \Delta_{m} \Psi_{1} & \\
= & \frac{2 \lambda}{\tau} \sum_{1}^{\prime}\left(-2 \pi q_{1}\right)^{n-m}(-\pi i)^{m} q_{1}^{m-1} \\
& \quad \times \cos m \phi_{q} e^{-2 \pi i q \cdot s} e^{-2 \pi q_{1}\left|x_{1}\right|}
\end{aligned}
$$

where

$\Delta_{m}=\left(\frac{\partial}{\partial \xi}\right)^{m}+\left(\frac{\partial}{\partial \eta}\right)^{m}, \quad \xi=x_{2}+i x_{3}, \quad \eta=x_{2}-i x_{3}$,

$\lambda= \begin{cases}1, & \text { if } x_{1}>0, \\ (-1)^{n-m}, & \text { if } x_{1}<0,\end{cases}$

$\phi_{q}=\tan ^{-1}\left(q_{3} / q_{2}\right)$.

The series in (16) converges rapidly for $\left|x_{1}\right|>h / 2$. Similar integration for $\Psi_{2}$ yields

$\Psi_{2}=\sum_{i}^{\prime} \frac{-1}{16 \pi \tau q_{1}^{3}}\left(2 \pi q_{1}\left|x_{1}\right|+1\right) e^{-2 \pi q_{1}\left|x_{1}\right|} e^{-2 \pi i q_{1} \cdot s}$.

Once again $\Psi_{2}$ given by (19) can be readily differentiated and the series for $\Psi_{2}$ thus obtained is useful for $\left|x_{1}\right|>h / 2$.

\section{B. The second method}

This method is useful for evaluating $\Psi_{1}$ and $\Psi_{2}$ for smaller values of $\left|x_{1}\right|$. Following Ewald's technique as in
Ishii $^{16}$ and Born and Misra, ${ }^{18}$ we obtain

$$
\Psi_{m}=\left[1 / \tau \Gamma(m)(-4 \pi)^{m-1}\right]\left(C_{\mathrm{I}}+C_{\mathrm{II}}+C_{\mathrm{III}}\right),
$$

where

$$
\begin{aligned}
C_{\mathrm{I}}= & \tau \rho^{m-3 / 2} \sum_{\mathrm{I}} \Phi_{-m+1 / 2}\left(\frac{\pi\left(\mathbf{r}-\mathbf{r}_{1}\right)^{2}}{\rho}\right), \\
C_{\mathrm{II}}= & \rho^{m} \int_{-\infty}^{\infty} d k e^{-2 \pi i k x_{1}} \int_{1}^{\infty} d \beta \beta^{m-1} \\
& \times \sum_{\mathrm{I}}^{\prime} e^{-\pi \beta\left(q_{\mathrm{l}}^{2}+k^{2}\right)-2 \pi i q_{\mathrm{l}} \mathbf{s}}, \\
C_{\mathrm{III}}= & \rho^{m-1 / 2} \Phi_{-m-1 / 2}\left(\pi x_{1}^{2} / \rho\right) .
\end{aligned}
$$

In the above expressions $\Phi$ is the incomplete gamma function and $\rho$ is an arbitrary constant whose value may be chosen so as to facilitate the subsequent calculations, a typical choice being $\rho=\pi h^{2}$. Although $C_{\mathrm{II}}$ can also be expressed in terms of a series in incomplete gamma functions, we have found it more convenient for the subsequent calculations to leave it as a double integral.

Now each term in (20) may be differentiated separately. To differentiate $C_{\mathrm{I}}$, we employ a theorem of Hobson ${ }^{19}$ according to which

$$
\begin{aligned}
& f_{n}\left(\frac{\partial}{\partial x_{1}}, \frac{\partial}{\partial x_{2}}, \frac{\partial}{\partial x_{3}}\right) F\left(r^{2}\right) \\
& \quad=\sum_{s=0}^{s<n / 2}\left(\frac{2^{n-2 s}}{s !} \frac{d^{n-2 s} F}{d\left(r^{2}\right)^{n-s}} \nabla^{2 s}\right) f_{n}\left(x_{1}, x_{2}, x_{3}\right)
\end{aligned}
$$

for any homogeneous polynomial function $f_{n}$ of degree $n$ in $x_{1}, x_{2}$, and $x_{3}$. This yields

$$
\begin{aligned}
\left(\frac{\partial}{\partial x_{1}}\right)^{n-\lambda} \Delta_{\lambda} C_{\mathrm{I}} & \\
= & 2 \tau \rho^{m-3 / 2} \sum_{1} \sum_{s=0}^{2 s<n-\lambda}\left(2 x_{1}\right)^{n-2 s-\lambda} \\
& \times \frac{(-1)^{n+s}(n-\lambda) !}{s !(n-2 s-\lambda) !}\left(\frac{\pi}{\rho}\right)^{n-s} \\
& \times \Phi_{n-s-m-1 / 2}\left(\frac{\pi\left(\mathbf{r}-\mathbf{r}_{1}\right)^{2}}{\rho}\right) R_{1}^{\lambda} \cos \lambda \phi_{1},
\end{aligned}
$$

where

$$
x_{2}-x_{21}=R_{1} \cos \phi_{1}, \quad x_{3}-x_{31}=R_{1} \sin \phi_{1} .
$$

Note that for $x_{1}=0$, only the term with $2 s=n-\lambda$ is nonzero.

It does not appear possible to reduce $C_{\mathrm{II}}$ to a simple summation over $l$ after its differentiation for an arbitrary $x_{1}$. However, since we are primarily interested in using this method for small $\left|x_{1}\right|$, we may first differentiate $C_{\mathrm{II}}$ with respect to $x_{1}$ and then expand $\exp \left(-2 \pi i k x_{1}\right)$ in a Taylor series near $x_{1}=0$. The resulting series is then termwise integrable yielding

$$
\begin{aligned}
& \left(\frac{\partial}{\partial x_{1}}\right)^{n-\lambda} \Delta_{\lambda} C_{\mathrm{II}} \\
& \quad=\sum_{1}^{\prime} 2 q_{1}^{\lambda} \cos \lambda \phi_{q_{1}} e^{-2 \pi i q_{1}-3}(-2 \pi)^{n-\lambda}(-\pi)^{\lambda} i^{n} \rho^{m-1 / 2}
\end{aligned}
$$




$$
\begin{aligned}
& \times \sum_{p=0}^{p=\infty} \frac{(-1)^{p}\left(2 \pi x_{1}\right)^{2 \rho}(2 p+n-1-\lambda) ! !}{(2 p) !(2 \pi \rho)^{p+(n-\lambda) / 2}} \\
& \times \Phi_{m-p-(n-\lambda) / 2-3 / 2}\left(\pi \rho q_{1}^{2}\right)
\end{aligned}
$$

for even values of $n-\lambda$; the result of differentiation is zero for odd values of $n-\lambda$. We note that the infinite series in $p$ reduces to a single term corresponding to $p=0$ when $x_{1}$ equals zero, whereas for $x_{1} \neq 0$, the above series in $x_{1}$ converges only for $\left|x_{1}\right|<h$.

To differentiate $C_{\mathrm{III}}$, the singular part in $\Phi_{-m-1 / 2}$ at $x_{1}=0$ is first extracted from it and the regular part is then expanded in a Taylor series. Thus we use the expansion

$$
\begin{aligned}
\Phi_{-m-1 / 2}\left(y^{2}\right)= & \Gamma\left(\frac{1}{2}-m\right)|y|^{2 m-1} \\
& -\sum_{p=0}^{p=\infty} \frac{(-1)^{p} y^{2 p}}{p !\left(p-2 m-\frac{1}{2}\right)},
\end{aligned}
$$

$$
y^{2} \equiv \pi x_{1}^{2} / \rho,
$$

in which the first term on the right-hand side (rhs) is singular at $\left|x_{1}\right|=0$. Since most of the calculations in which we are presently interested require the evaluation of the regular part of $\Psi_{1}$ or $\Psi_{2}$ near $r=0$, we may ignore the first term and differentiate only the second term. This yields

$$
\begin{aligned}
\left(\frac{\partial}{\partial x_{1}}\right)^{n} C_{\mathrm{III}}^{*}= & -\rho^{m-1 / 2} \sum_{2 p=n}^{\infty}\left(\frac{-\pi}{\rho}\right)^{p} \\
& \times \frac{(2 p) ! x_{1}^{2 p-n}}{(2 p-n) ! p !\left(p-m+\frac{1}{2}\right)},
\end{aligned}
$$

where $C_{\text {III }}^{*}$ denotes the regular part of $C_{\mathrm{III}}$. Once again, only one term, with $2 p=n$ in (29) remains nonzero when $x_{1}=0$. In case it is necessary to evaluate the derivatives of $C_{\text {III }}$ for nonzero but small $\left|x_{1}\right|$, we may use the following formula obtained by a direct differentiation of $C_{\mathrm{III}}$ :

$$
\begin{aligned}
\left(\frac{\partial}{\partial x_{1}}\right)^{n} C_{\mathrm{III}}= & \rho^{m-1 / 2} \sum_{p=0}^{p<n / 2} x_{1}^{n-2 p} \frac{2^{n-2 p} n !}{p !(n-2 p) !} \\
& \times\left(\frac{-\pi}{\rho}\right)^{n-p} \Phi_{-m+n-p-1 / 2}\left(\frac{\pi x_{1}^{2}}{\rho}\right) .
\end{aligned}
$$

\section{The third method}

This method is suitable for evaluating higher-order derivatives of $\Psi_{m}$. Combining the Green's function for the Laplace equation in an infinite domain with (11), we obtain

$$
\Psi_{1}(\mathbf{r})=\sum_{i}\left(\frac{1}{\left|\mathbf{r}-\mathbf{r}_{1}\right|}-\frac{1}{\tau} \int_{\tau_{1}} \frac{d \mathbf{r}^{\prime}}{\left|\mathbf{r}-\mathbf{r}^{\prime}\right|}\right),
$$

where $\mathbf{r}^{\prime}$ is the variable of area integration in the lth unit cell. Note that although the sum over all 1 does not converge individually for each term on the rhs of (31), the difference between the two must converge since $\Psi_{1}$ is a finite function. It may be also noted that the second term on the rhs of (31) is a Laplace function of $\left|x_{1}\right|$ and hence it must be of the form $c_{1}\left|x_{1}\right|+c_{2}$, where $c_{1}$ and $c_{2}$ are constants with $\left|c_{2}\right| \rightarrow \infty$ as more terms in the sum are included. Since we are interested in using this method to evaluate only the higher-order derivatives of $\Psi_{1}$ and $\Psi_{2}$, we note that the derivatives of the second term on the rhs of (31) vanish identically yielding the following formulas:

$$
\begin{array}{ll}
\left(\frac{\partial}{\partial x_{1}}\right)^{n} \Psi_{1}(\mathbf{r})=\sum_{1}\left(\frac{\partial}{\partial x_{1}}\right)^{n} \frac{1}{\left|\mathbf{r}-\mathbf{r}_{1}\right|}, & n \geqslant 2, \\
\left(\frac{\partial}{\partial x_{1}}\right)^{n} \Psi_{2}(\mathbf{r})=\sum_{1}\left(\frac{\partial}{\partial x_{1}}\right)^{n} \frac{\left|\mathbf{r}-\mathbf{r}_{1}\right|}{2}, & n \geqslant 4 .
\end{array}
$$

The restrictions on $n$ in the above formulas are determined from the requirement that the sums in them must converge. In practice, $\Psi_{m}$ calculated from the above equations converges rapidly for $n \geqslant 8$. This method is also useful in verifying the accuracy of the formulas derived by the first two methods.

\section{SHEAR FLOW}

\section{A. The statement of the problem and the method of solution}

As a first application of the planar singular solutions described in Sec. II, we consider the problem of determining the velocity field near the surface of a porous medium. Thus we suppose that the spherical particles of radius $a$ are held fixed with their centers at

$$
\mathbf{r}=\mathbf{r}^{\alpha}+\mathbf{r}^{\prime}, \quad \alpha=1,2,3, \ldots .
$$

The model of the porous medium is shown in Fig. 1. The fluid motion satisfies the Stokes equations of motion plus the following boundary conditions:

$$
\begin{aligned}
& u_{i} \rightarrow 0, \text { as } x_{1} \rightarrow \infty, \\
& \frac{\partial u_{i}}{\partial x_{j}}-\gamma \delta_{i 2} \delta_{j 1}, \quad \text { as } x_{1} \rightarrow-\infty, \\
& u_{i}=0, \text { on the spheres, } \\
& u_{i}(\mathbf{r})=u_{i}\left(\mathbf{r}+\mathbf{r}_{1}\right) .
\end{aligned}
$$

The solution of the above problem can be expressed as

$$
u_{i}(\mathbf{r})=\sum_{\alpha=1}^{\alpha=\infty} \sum_{n=0}^{n=\infty} F_{j k l \cdots n}^{\alpha} \frac{\partial^{n} v_{i j}\left(\mathbf{r}-\mathbf{r}^{\alpha}\right)}{\partial x_{k} \partial x_{l} \cdots \partial x_{n}}+u_{i}^{*}\left(x_{1}\right),
$$

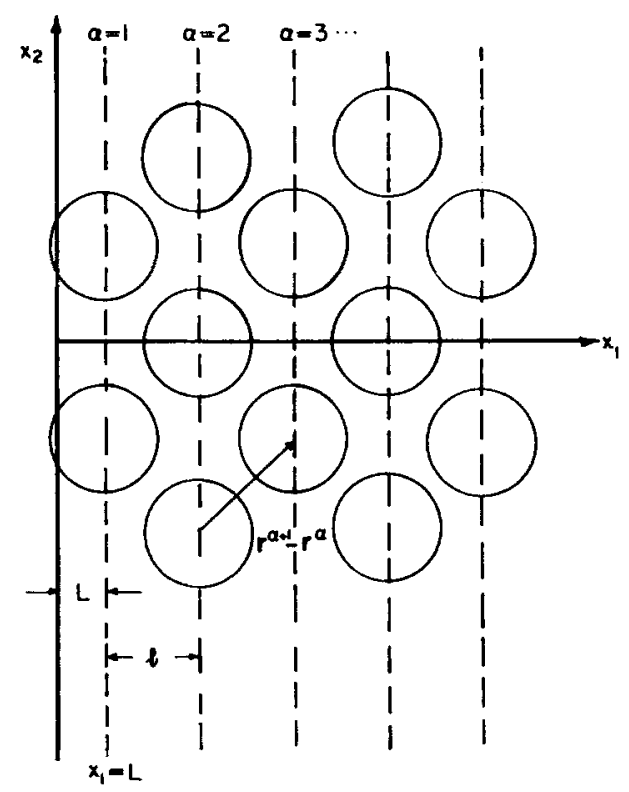

FIG. 1. A sketch of a semi-infinite array of spheres. 
where $u_{i}^{*}$ will be presently specified and $\mathrm{F}^{\alpha}$ is an unknown referred to as the strength of a singularity. Owing to linearity of the governing equations, we must have that

$$
\mathbf{F}_{(n)}^{\alpha}=\mathbf{\Lambda}_{(n+2)}^{\alpha}: \gamma_{(2)}, \quad \text { with }\left(\gamma_{(2)}\right)_{i j}=\gamma \delta_{i 2} \delta_{j 1},
$$

where $\Lambda_{(n+2)}$ is a tensor of rank $n+2$ that depends only on the geometry of the array. We shall restrict our attention to the semi-infinite cubic arrays, for which the tensor $\Lambda$ must be a function of the orientation of the array, which is taken to be along the $x_{1}$ axis, and must have components that remain unchanged upon a $90^{\circ}$ rotation of the $x_{2}$ and $x_{3}$ axes in the $x_{1}$ plane. Upon using the most general forms of the tensors satisfying the above two conditions and rearranging, we finally arrive at

$$
\begin{aligned}
u_{1}= & u_{1}^{*}+\sum_{\alpha}\left[\mathbf{G}^{\alpha}\left(-\frac{\partial^{2} \Psi_{2}}{\partial x_{1} \partial x_{2}}\right)\right. \\
& \left.+\mathbf{H}^{\alpha}\left(\frac{\partial \Psi_{1}}{\partial x_{2}}\right)-\mathbf{L}^{\alpha}\left(\frac{\partial^{2} \Psi_{1}}{\partial x_{1} \partial x_{2}}\right)\right], \\
u_{2}= & u_{2}^{*}+\sum_{\alpha}\left[\mathbf{G}^{\alpha}\left(\Psi_{1}-\frac{\partial^{2} \Psi_{2}}{\partial x_{2}^{2}}\right)\right. \\
& \left.-\mathbf{H}^{\alpha}\left(\frac{\partial \Psi_{1}}{\partial x_{1}}\right)-\mathbf{L}^{\alpha}\left(\frac{\partial^{2} \Psi_{1}}{\partial x_{2}^{2}}\right)\right], \\
u_{3}= & u_{3}^{*}+\sum_{\alpha}\left[\mathbf{G}^{\alpha}\left(-\frac{\partial^{2} \Psi_{2}}{\partial x_{2} \partial x_{3}}\right)-\mathbf{L}^{\alpha}\left(\frac{\partial^{2} \Psi_{1}}{\partial x_{2} \partial x_{3}}\right)\right],
\end{aligned}
$$

where

$$
\mathbf{G}^{\alpha}=\sum_{n=0}^{n=\infty} \sum_{m=0}^{m<n / 2} A_{n m}^{\alpha}\left(\frac{\partial}{\partial x_{1}}\right)^{n-m} \Delta_{m}
$$

and $\mathbf{H}^{\alpha}$ and $\mathbf{L}^{\alpha}$ are defined similarly with $A_{n m}^{\alpha}$ in the above equation replaced, respectively, by $B_{n m}^{\alpha}$ and $C_{n m}^{\alpha}$, the constants $A_{n m}^{\alpha}, B_{n m}^{\alpha}$, and $C_{n m}^{\alpha}$ being zero for odd integral values of $m$. In (38)-(40) $u_{i}$ is evaluated at $\mathbf{r}$ while $\Psi_{1}$ and $\Psi_{2}$ are evaluated at $\mathbf{r}-\mathbf{r}^{\alpha}$.

Now we determine $u_{i}^{*}$ from the boundary conditions for $u_{i}$ at $x_{1}= \pm \infty$ and from the requirement that the velocity field must be a continuous and differentiable function at all points in the fluid. The latter condition, in particular, requires that

$$
\begin{aligned}
\nabla^{2} u_{2}= & \nabla^{2} u_{2}^{*}+\sum_{\alpha} \nabla^{2}\left[\mathbf{G}^{\alpha}\left(\Psi_{1}-\frac{\partial^{2} \Psi_{2}}{\partial x_{2}^{2}}\right)\right. \\
& \left.-\mathbf{H}^{\alpha}\left(\frac{\partial \Psi_{1}}{\partial x_{1}}\right)-\mathbf{L}^{\alpha}\left(\frac{\partial^{2} \Psi_{1}}{\partial x_{2}^{2}}\right)\right]
\end{aligned}
$$

must also be continuous everywhere in the fluid. Clearly since the terms in the parentheses on the rhs of the above equation are singular on the $x_{1}=x_{1}^{\alpha}$ planes, the function $u_{2}^{*}$ must also be singular so that it cancels these singularities identically. Thus on noting that, for any $r$ inside the fluid [ $\mathrm{cf}$. (11) ],

$$
\begin{aligned}
& \nabla^{2} \Psi_{1}\left(\mathbf{r}-\mathbf{r}^{\alpha}\right)=4 \pi / \tau \delta\left(x_{1}-x_{1}^{\alpha}\right), \\
& \nabla^{2}\left(\left|x_{1}\right|\right)=2 \delta\left(x_{1}-x_{1}^{\alpha}\right),
\end{aligned}
$$

we obtain, after adding up to linear terms in $x_{1}$, the following expression for $u_{2}^{*}$ :

$$
\begin{aligned}
u_{2}^{*}= & b_{1} x_{1}+b_{2}-\sum_{\alpha} \frac{2 \pi}{\tau} \mathbf{G}^{\alpha}\left|x_{1}-x_{1}^{\alpha}\right| \\
& +\sum_{\alpha} \frac{2 \pi}{\tau} \mathbf{H}^{\alpha} \operatorname{sgn}\left(x_{1}-x_{1}^{\alpha}\right),
\end{aligned}
$$

where $\operatorname{sgn}(x)$ is +1 for $x>0$ for $x=0$, and -1 for $x<0$. Now it is easy to show [cf. (13)] that $\Psi_{m}\left(r-r^{\alpha}\right)$ approaches zero exponentially as $\left|x_{1}-x_{1}^{\alpha}\right| \rightarrow \infty$ and hence, provided that $\mathbf{G}^{\alpha}$ and $\mathbf{H}^{\alpha}$ also tend to zero sufficiently rapidly, we may ignore the terms containing $\Psi_{1}$ and $\Psi_{2}$ in (42) and satisfy the boundary conditions for $u_{2}$ at $x_{1}= \pm \infty$ simply by considering $u_{2}^{*}$ alone. Thus, on noting that $\Delta_{0}=2$, (45) yields

$$
\begin{aligned}
& -\gamma=b_{1}+\frac{4 \pi}{\tau} \sum_{\alpha} A_{00}^{\alpha}, \\
& 0=b_{1}-\frac{4 \pi}{\tau} \sum_{\alpha} A_{00}^{\alpha}, \\
& 0=b_{2}+\frac{4 \pi}{\tau} \sum_{\alpha}\left(x_{1}^{\alpha} A_{00}^{\alpha}-A_{10}^{\alpha}+B_{00}^{\alpha}\right) .
\end{aligned}
$$

From (46) and (47) we obtain

$b_{1}=\frac{4 \pi}{\tau} \sum_{\alpha} A_{\mathrm{oO}}^{\alpha}=\frac{-\gamma}{2}$,

$u_{2}=-\gamma\left(x_{1}-h U_{s}\right)+$ e.d.t. as $x_{1} \rightarrow \infty$,

$h \gamma U_{s}=2 b_{2}=\frac{-8 \pi}{\tau} \sum_{\alpha}\left(x_{1}^{\alpha} A_{00}^{\alpha}-A_{10}^{\alpha}+B_{00}^{\alpha}\right)$,

where e.d.t. stands for the terms that decay to zero exponentially as $x_{1} \rightarrow-\infty$. The term $U_{s}$, which depends only on the geometry of the array and the volume fraction of the spheres in the array, will be referred to as the slip coefficient of the array.

The result (49) can be interpreted as follows. First of all, it is easy to show that the constant $A_{00}^{a}$ is related to the drag force $F^{\alpha}$ on the spheres in the $x_{1}=x_{1}^{\alpha}$ plane by a simple relationship $F^{\alpha}=8 \pi \mu A_{00}^{\alpha}$, where $\mu$ is the viscosity of the fluid. Thus the second equality in (49) is equivalent to

$$
\mu \gamma \tau=\sum_{\alpha} F^{\alpha}
$$

or, in other words, the sum total of the frictional forces exerted on the solids in the porous medium per unit cross-sectional area is equal to the shear stress in the fluid at $x_{1}=-\infty-$ a result that can also be derived from the principle of overall conservation of momentum.

Returning to the choice of $u_{i}^{*}$, we observe that since each of the terms associated with $\Psi_{1}$ or $\Psi_{2}$ in (38) and (40) involves a differentiation with respect to $x_{2}$ or $x_{3}$, they are well behaved at all points in the fluid and, consequently, $u_{1}^{*}$ $=u_{3}^{*}=0$.

The expressions (38)-(40), (45), (49), and (51) together represent the formal solution for the velocity field. This solution satisfies all the conditions listed in (35) except for the no-slip boundary condition on the surface of the spheres, which we consider next. From experience with similar problems in the past, we know that it is best to satisfy the no-slip boundary condition on the spheres by expanding the velocity field in terms of spherical harmonics near their 
centers. Accordingly, we first express $u_{i}$ near the center of a sphere located on the $x_{1}=x_{1}^{\alpha}$ plane as a sum of singular and regular terms:

$$
u_{i}=u_{i, \alpha}^{s}+u_{i, \alpha}^{r},
$$

where $u_{i, \alpha}^{s}$ is singular and $u_{t, \alpha}^{r}$ is regular at $\mathbf{r}=\mathbf{r}^{\alpha}$. Clearly the terms containing $A_{n m}^{\alpha}, B_{n m}^{\alpha}$, and $C_{n m}^{\alpha}$ in the general solution contribute to $u_{i, \alpha}^{s}$ and the remainder to $u_{i, \alpha}^{r}$. Next, we expand the regular part of $u_{i}$ in spherical harmonics by using a result given in Ref. 8, according to which the coefficient of $Y_{n}^{m}$ [cf. (55)] is given by

$$
\begin{gathered}
\epsilon_{m}\left[\left(\frac{\partial}{\partial x_{1}}\right)^{n-m} \Delta_{m}-C\left(\frac{\partial}{\partial x_{1}}\right)^{n-m-2} \Delta_{m} \nabla^{2}\right. \\
\left.+\frac{r^{2}}{4 n+6}\left(\frac{\partial}{\partial x_{1}}\right)^{n-m} \Delta_{m} \nabla^{2}\right] u_{i, \alpha}^{r}(\mathbf{r}),
\end{gathered}
$$

with

$$
\begin{aligned}
& \epsilon_{m}= \begin{cases}\frac{1}{2}, & m=0, \\
(-2)^{m}, & m \neq 0,\end{cases} \\
& C= \begin{cases}{[1 /(4 n-2)][(n-m) ! /(n-m-2) !],} & n-m \geqslant 2, \\
0, & \text { otherwise, }\end{cases}
\end{aligned}
$$$$
(n+m) ! Y_{n}^{m}=r^{n} P_{n}^{m}(\cos \theta) \cos m \phi \text {. }
$$

Here $r, \theta$, and $\phi$ are the spherical polar coordinates measured from the center of the sphere on the $x_{1}=x_{1}^{\alpha}$ plane.

This completes the description of the formal solution for the velocity field. The infinite series in $\alpha, n$, and $m$ in this solution can be suitably truncated and, by solving the equations that result upon application of the no-slip boundary condition to the spheres on each of the planes, the velocity field and $U_{s}$ can be determined numerically. Before we present the numerical results, however, we shall analyze analytically the case of dilute arrays, i.e., $a \ll h$.

\section{B. The low volume fraction asymptote}

In this limit we anticipate that the flow field will be adequately approximated even if we retain only one singularity per particle. Accordingly we retain the force singularity (the $A_{00}$ term) for each particle and equate to zero the coefficient of $Y_{0}^{0}$ in the expansion of $u_{2}$ around the center of each particle. Thus the velocity field is approximated as

$$
\begin{aligned}
u_{2}(\mathbf{r})= & -\frac{1}{2 \mu \tau} \sum_{\alpha=1}^{\infty} F^{\alpha}\left(x_{1}-x_{1}^{\alpha}-\left|x_{1}-x_{1}^{\alpha}\right|\right) \\
& +\sum_{\alpha=1}^{\infty} \frac{F^{\alpha}}{4 \pi \mu}\left(\Psi_{1}-\frac{\partial^{2} \Psi_{2}}{\partial x_{2}^{2}}\right)\left(\mathbf{r}-\mathbf{r}^{\alpha}\right)
\end{aligned}
$$

where we have used the fact that $F^{\alpha}=8 \pi \mu A_{00}^{\alpha}$. Next, we note that, in view of (11) and (12), $\Psi_{1}$ and $\Psi_{2}$ must have the following expansions near their singularities:

$$
\begin{aligned}
& \Psi_{1}=1 / r+2 \pi / \tau\left|x_{1}\right|+\text { const }+O\left(r^{4}\right), \\
& \Psi_{2}=r / 2+\pi / 3 \tau\left|x_{1}^{3}\right|+\text { const }+O\left(r^{4}\right) .
\end{aligned}
$$

On equating to zero the coefficient of $Y_{0}^{0}$ in the expansion of $u_{2}$ near a sphere on the $x_{1}=x_{1}^{m}$ plane, we obtain

$0=-\frac{1}{2 \mu \tau} \sum_{\alpha=1}^{m-1}(m-\alpha) l F^{\alpha}+\frac{F^{m}}{6 \pi a \mu}+\sum_{\alpha=1}^{\infty} \frac{F^{\alpha}}{4 \pi \mu} \varphi_{m \alpha}$, where $\varphi_{m a}$ is the regular part of $\Psi_{1}-\partial^{2} \Psi_{2} / \partial x_{2}^{2}$ evaluated at $\mathbf{r}^{m}-\mathbf{r}^{\alpha}$. [Note that the singular part of the latter quantity arises only for $m=\alpha$ and the term $F^{m} / 6 \pi \mu a$ in (59) represents the contribution of this singular term.] The relation (59) is valid for all positive integers $m$, and provides a sufficient condition for determining $F^{m}$ to the leading-order approximation as $a \rightarrow 0$. At first, it might appear from (59) that this leading-order term is $O(a)$. However, we recall that $\Sigma F^{m}=\gamma \mu \tau$ [cf. (52)], which implies that the sum of the $F$ 's is an $O$ (1) quantity. Thus a straightforward perturbation scheme cannot be employed to determine the $F$ 's. Since as $a \rightarrow 0$ we expect the slip velocity to become very large and the velocity to decay slowly to zero as $x_{1} \rightarrow \infty$, we seek the solution of (59) in the form

$$
F^{\alpha}=B e^{-\alpha \sigma(a)},
$$

with the anticipation that $\sigma \rightarrow 0$ as $a \rightarrow 0$. Substituting (60) into (52), we obtain

$$
B=\gamma \mu \tau\left(1-e^{-\sigma}\right)=\gamma \mu \tau\left[\sigma+O\left(\sigma^{2}\right)\right] .
$$

Now $\varphi_{m \alpha}$ decays exponentially with the increase in $|m-\alpha|$ independently of $a$ and hence we may neglect the third term on the rhs of (59) with a relative error of $O(\sigma)$. Substituting (60) into the remainder of (59) then yields a leading-order approximation for $\sigma$ :

$$
\sigma^{2}=6 \pi a l / \tau
$$

where $l$ is the minimum distance between two successive $x_{1}$ planes on which the centers of the spheres are located (cf. Fig. 1). Combining (61) and (62) we obtain the following approximation for $F^{\alpha}$ :

$$
F^{\alpha}=\mu \tau \gamma(6 \pi a l / \tau)^{1 / 2} e^{-\alpha(6 \pi a l / r)^{1 / 2}} .
$$

The slip coefficient $U_{s}$ is given by

$$
h U_{s}=\sum_{1}^{\infty} \frac{x_{1}^{\alpha} F^{\alpha}}{\mu \gamma}=\left(\frac{\tau l}{6 \pi a}\right)^{1 / 2},
$$

where we have chosen $L \equiv x_{1}^{1}=0$ (cf. Fig. 1). Let us now express the above results in terms of the permeability of the array. In this limit of infinite dilution, $a \ll h$, the permeability is given by

$$
k_{\infty}=\tau l / 6 \pi a,
$$

so that the slip coefficient is given by

$$
h U_{s}=\sqrt{k_{\infty}} .
$$

This result for very dilute arrays was also given by Saffman. ${ }^{20}$ Since to this order of approximation $F_{\alpha}$ equals the Stokes drag $6 \pi \mu U^{\alpha} a$, where $U^{\alpha}$ is the mean velocity of the fluid in the neighborhood of $x_{1}=x_{1}^{\alpha}$, independently of the detailed arrangement of the particles, it is not surprising that our result agrees with that given by Saffman. We also note that the ratio of the drag forces on the spheres in successive planes is given by

$$
R \equiv F^{\alpha+1} / F^{\alpha}=e^{-(6 \pi a l / \tau)^{1 / 2}}=e^{-1 / \sqrt{k_{\infty}}},
$$

which is in agreement with the estimate first given by Brinkman ${ }^{17}$ who derived it by invoking an ad hoc approximation.

Although the results derived here merely confirm the analyses of the previous investigators, it must be appreciated that the derivation has followed a different approach than in 
the past and is capable of extension to higher orders in $a / h$. Thus, for example, at the next order of approximation the geometry of the array becomes important and $k_{\infty}$ in (67) is now replaced by the estimate of the permeability to $O\left(\phi^{1 / 3}\right)$ as given by Hasimoto. ${ }^{21}$ Here $\phi$ is the volume fraction of the spheres as given by

$$
\phi=4 \pi a^{3} / 3 \tau l \text {. }
$$

We shall, however, not pursue the asymptotic analysis any further (mainly because it is easier to evaluate $U_{s}, R$, or the velocity distribution numerically for the complete range of volume fraction of the spheres), except for noting that, on examining the nature of the higher-order terms, we find that

$$
A_{n+2, m}^{\alpha}, B_{n+1, m}^{\alpha} \text {, and } C_{n m}^{\alpha}=O\left(a^{2 n+5}\right) A_{00}^{\alpha} \text {. }
$$

\section{Numerical results}

To determine numerically the velocity field for nondilute arrays we must first truncate the infinite series for the differential operators $\mathbf{G}^{\alpha}, \mathbf{H}^{\alpha}$, and $\mathbf{L}^{\alpha}$, as well as the series for $\alpha$ in the formal solution for the velocity field presented in Sec. III A. The asymptotic analysis presented in Sec. III B provides us with a systematic procedure for doing this. Thus all the constants $A_{n m}$ with $n \geqslant N_{p}, B_{n m}$ with $n \geqslant N_{p}-1$, and $C_{n m}$ with $n \geqslant N_{p}-2$, where $N_{p}$ is a suitably chosen integer, were neglected and the no-slip boundary condition on the spheres was applied to generate the equations among the constants retained by considering the coefficients of $Y_{n}^{2 m}$ in $u_{2}, Y_{n}^{2 m+1}$ in $u_{1}$, and $Y_{n}^{* 2 m}$ in $u_{3}$ with $n<N_{p}$. Here $Y_{n}^{* m}$ is a spherical harmonic function with the cosine term in (55) replaced by a sine term. Also, instead of applying the no-slip boundary condition on all the planar arrays, only those with $\alpha \leqslant N_{t}$, where $N_{t}$ is another suitably chosen integer, were selected for satisfying the no-slip boundary condition. This is equivalent to setting all the constants $A_{n m}, B_{n m}$, and $C_{n m}$ for $\alpha>N_{t}$ to zero. Alternatively, it may be assumed that all the constants for $\alpha>N$, are related to the corresponding constants with $\alpha=N_{t}$ by a simple decay factor to be determined numerically by a trial-and-error procedure. The latter scheme is slightly more complicated to implement and it did not offer any special advantage except at very small volume fractions. Both $N_{p}$ and $N$, were increased systematically for each selected value of $a / h$ until no significant change in $U_{s}$ was observed. As one would expect, smaller $a / h$ required smaller $N_{p}$ and greater $N$, whereas larger $a / h$ required smaller $N_{t}$ and greater $N_{p}$. A three-digit accuracy was obtained in most cases. We may add here that the application of the no-slip boundary condition on the spheres required an accurate evaluation of the various derivatives of $\Psi_{1}$ and $\Psi_{2}$ at the vector difference $\mathbf{r}^{\alpha+m}-\mathbf{r}^{\alpha}$ for various $m$ and these were evaluated by the methods described in Sec. II for the three cubic arrays separately and stored in the computer for repeated use. A typical run for a given $a / h$ took anywhere from $10 \mathrm{sec}$ to $1 \mathrm{~min}$ of CPU time on an IBM 4341 computing machine; thus the method described here was found to be very efficient.

Before we present the results of the numerical computations, a few more points need to be discussed. First, the results will be presented in terms of a nondimensional radius $\chi$ defined by

$$
\chi=a / h=\left(\phi / \phi_{m}\right)^{1 / 3},
$$

where $\phi_{m}$ is the maximum allowable volume fraction at which the spheres begin to touch their neighbors. Its value along with various other parameters, such as the basis vectors of the lattice, $l$ and $\tau$, for the three cubic arrays, the simple cubic (SC), the body-centered cubic (BCC), and the face-centered cubic (FCC), are given in Table $I$.

An important result of our study is the ability to determine the detailed velocity field near porous surfaces. However, the three-dimensional velocity profiles are rather tedious to report and hence we shall restrict ourselves here to only the discussion of the numerical results for $U_{s}$ and $\boldsymbol{R}$ defined in Secs. III A and III B. As mentioned in Sec. I, and also by Larson and Higdon ${ }^{14,15}$ and Saffman, ${ }^{20}$ a change in the definition of the position of the nominal "interface" by a distance comparable to the microscale length $h$ will produce an $O(1)$ change in $U_{s}$ when the permeability of the medium is not very large or, equivalently, when $a / h$ is not small $(\sqrt{k} \ngtr h)$. In practice, however, it is seldom possible to define precisely the location of the "interface," as the microscale is usually very small. Thus admittedly, from the practical standpoint, the determination of $U_{s}$ at very high volume fractions is perhaps not of great significance. On the other hand, as mentioned in Sec. $I$, there is considerable interest in assessing the accuracy of the continuum approximate theories proposed in the literature as far as their application to the problems in which the length scale relevant to the macroscopic property ( $\sqrt{k}$ in the present case) and the microscale are of comparable magnitude. In this sense, the results for large volume fractions provide the most stringent tests for the approximate theories, and thus are quite useful.

To make the comparison between the exact results and the approximate theories meaningful at large volume fractions, we must define the location of the "interface." We have chosen the "interface" so that the first row of spheres are located at a distance $L=l / 2$ (cf. Fig. 1), mainly to ensure that each sphere in the porous medium can be regarded to be exactly at the center of a three-dimensional unit cell of width $l$. This choice of $L$ is different from the one employed in Sec. II B, viz., $L=0$, but it can be easily confirmed that the leading-order approximations derived there remain valid independently of the choice of $L$ as long as $L \ll \sqrt{k}$.

Finally, we also wish to note that Larson and Higdon ${ }^{14,15}$ defined the nominal interface by choosing $L=0$ and then employed two different definitions for the slip coefficient based on the volumetric flux of the fluid both above and below the nominal interfaces (denoted in their papers by

TABLE I. Parameters of the cubic arrays. SC: simple cubic; BCC: bodycentered cubic; and FCC: face-centered cubic.

\begin{tabular}{cccc}
\hline & SC & BCC & FCC \\
\hline $\mathbf{r}^{\alpha+1}-\mathbf{r}^{\alpha}$ & $(2,0,0) h$ & $2 / \sqrt{ } 3(1,1,1) h$ & $\sqrt{ } 2(1,1,0) h$ \\
$\mathbf{a}_{2}$ & $(0,2,0) h$ & $4 / \sqrt{ } 3(0,1,0) h$ & $\sqrt{ } 2(0,1,1) h$ \\
$\mathbf{a}_{3}$ & $(0,0,2) h$ & $4 / \sqrt{ } 3(0,0,1) h$ & $\sqrt{ } 2(0,1,-1) h$ \\
$l / h$ & 2 & $2 / \sqrt{ } 3=1.155$ & $\sqrt{ } 2=1.414$ \\
$\tau / h^{2}$ & 4 & $16 / 3=5.333$ & 4 \\
$\phi_{m}$ & $\pi / 6=0.524$ & $\sqrt{ } 3 \pi / 8=0.680$ & $\pi / 3 \sqrt{ } 2=0.740$ \\
\hline
\end{tabular}


$Q^{+}$and $Q^{-}$), whereas we have chosen the far-field $\left(x_{1} \rightarrow-\infty\right)$ behavior of $u_{2}$ to define $U_{s}$. Since the averages of $\Psi_{1}$ and $\Psi_{2}$ over any (two-dimensional) unit cell in an $x_{1}$ plane not passing through the particles are zero,

$$
\left\langle u_{2}\right\rangle\left(x_{1}\right)=\gamma\left(-x_{1}+h U_{s}\right)
$$

for any $x_{1}$ less than zero if no sphere passes through the $x_{1}$ plane. Thus the definitions of the slip coefficient based on the far-field behavior of $u_{2}$ and $Q^{+}$are equivalent as long as $L$ is chosen such that the interface does not intersect the spheres.

The results of the numerical computations for $U_{s}$ and $R$ for various $\chi$ for the three cubic arrays are given in Table II under the heading of "exact" to distinguish them from the predictions of the approximate theories to be described in Sec. III D. For smaller values of $\chi$, less than approximately 0.6 , the ratio $R=F^{\alpha+1} / F^{\alpha}$ became nearly independent of $\alpha$ for $\alpha \geqslant 2$ and the reported values of $R$ correspond to these constant values. For larger $\chi$ for which $R$ varied considerably with $\alpha$, only the value corresponding to $\alpha=1$ is given; these are enclosed in parentheses to indicate that $R$ is dependent on $\alpha$.

We note that the coefficient $U_{s}$ remains positive for all $\chi$ in the case of a simple cubic array but does become negative at higher values of $\chi$ for the body-and face-centered cubic arrays. It should be noted that with our choice of the definition of the "interface," $L=l / 2$, there is a protrusion of the spheres beyond the interface plane for $\chi$ greater than $1 / \sqrt{3}$ $=0.577 \ldots$ and $1 / \sqrt{2}=0.707 \ldots$, respectively, for the bodyand face-centered cubic arrays. As pointed out by Larson and Higdon, ${ }^{14.15}$ such negative values of $U_{s}$ are, of course,

TABLE II. Results for the shear flow past semi-infinite cubic arrays. Br: Brinkman approximation; CA: Chang-Acrivos approximation.

\begin{tabular}{|c|c|c|c|c|c|c|c|}
\hline \multirow[b]{2}{*}{$\chi$} & \multicolumn{3}{|c|}{ Exact } & \multicolumn{2}{|r|}{$\mathrm{Br}$} & \multirow{2}{*}{$\frac{\mathrm{CA}}{U}$} & \multirow[b]{2}{*}{ Array } \\
\hline & $K$ & $U_{s}$ & $R$ & $U_{s}$ & $R$ & & \\
\hline 0.01 & 1.014 & 6.512 & 0.734 & 6.468 & 0.734 & $(6.8)$ & \\
\hline 0.03 & 1.029 & 3.770 & 0.644 & 3.680 & 0.581 & 4.3 & \\
\hline 0.05 & 1.076 & 2.925 & 0.490 & 2.808 & 0.491 & 3.19 & \\
\hline 0.20 & 1.388 & 1.467 & 0.196 & 1.238 & 0.198 & 1.57 & SC \\
\hline 0.50 & 2.842 & 0.830 & 0.024 & 0.547 & 0.026 & 0.94 & \\
\hline 0.80 & 10.05 & 0.441 & $\left(-1.1 \times 10^{-4}\right)$ & 0.230 & $1.6 \times 10^{-4}$ & 0.52 & \\
\hline 0.90 & 19.16 & 0.323 & $\ldots$ & 0.157 & $10^{-5}$ & 0.37 & \\
\hline 1.00 & 42.1 & 0.21 & $\ldots$ & 0.10 & $10^{-x}$ & 0.21 & \\
\hline 0.03 & 1.050 & 3.256 & 0.700 & 3.22 & 0.700 & 3.23 & \\
\hline 0.05 & 1.086 & 2.496 & 0.625 & 2.45 & 0.625 & 2.51 & \\
\hline 0.20 & 1.449 & 1.146 & 0.338 & 1.06 & 0.34 & 1.18 & \\
\hline 0.50 & 3.384 & 0.494 & 0.098 & 0.439 & 0.07 & 0.55 & $\mathrm{BCC}$ \\
\hline 0.60 & 5.108 & 0.348 & $(0.05)$ & 0.326 & 0.03 & 0.397 & \\
\hline 0.80 & 16.85 & 0.085 & $\left(8 \times 10^{-3}\right)$ & 0.16 & $6 \times 10^{-4}$ & 0.075 & \\
\hline 0.90 & 42.8 & -0.04 & $\left(-6 \times 10^{-3}\right)$ & 0.09 & $\cdots$ & -0.1 & \\
\hline 1.001 & 163 & -0.16 & $\ldots$ & 0.05 & $\cdots$ & -0.27 & \\
\hline 0.05 & 1.088 & 2.418 & 0.548 & 2.348 & 0.548 & 2.44 & \\
\hline 0.20 & 1.467 & 1.144 & 0.248 & 1.02 & 0.249 & 1.20 & \\
\hline 0.50 & 3.575 & 0.538 & 0.040 & 0.41 & 0.032 & 0.62 & \\
\hline 0.60 & 5.538 & 0.400 & $\left(1.6 \times 10^{-2}\right)$ & 0.300 & $9 \times 10^{-3}$ & 0.47 & $\mathrm{FCC}$ \\
\hline 0.80 & 20.9 & 0.149 & $\left(-3 \times 10^{-3}\right)$ & 0.134 & $2 \times 10^{-5}$ & 0.156 & \\
\hline 0.90 & 65.1 & 0.03 & $\left(-4 \times 10^{-3}\right)$ & 0.07 & $3 \times 10^{-4}$ & -0.02 & \\
\hline 1.004 & 450 & -0.1 & $\left(-10^{-2}\right)$ & 0.026 & $\ldots$ & -0.19 & \\
\hline
\end{tabular}

plausible at higher values of $\chi$. We also note that at higher values of $\chi$, greater than, say, 0.8 , the ratio of the drag forces on the first two rows of spheres becomes negative, indicating the presence of regions of reverse flow.

To compare the numerical results with the asymptotic formulas derived in Sec. III C, we first note that the permeability of the porous medium is related to the infinite-dilution permeability by a correction factor $K$, which is defined as the ratio of the drag force on a sphere in an infinite array to the Stokes drag, by a simple expression

$$
k=k_{\infty} / K, \quad \text { with } K \equiv F / 6 \pi \mu U a .
$$

As mentioned in Sec. III C, an analysis of the next to leading-order approximations for $U_{s}$ and $R$ essentially replaces $k_{\infty}$ by the estimate of $k$ provided by Hasimoto, ${ }^{21}$ according to which

$$
K=(1+\lambda \chi) \quad \text { (Hasimoto), }
$$

where $\lambda$ equals $1.4168,1.5758$, and 1.6210 for, respectively, the simple, body-centered, and face-centered cubic arrays. A comparison of the numerical results with the asymptotic relations of Sec. III $\mathrm{C}$ with this slight correction for the permeability shows an agreement within $5 \%$ for $U_{s}$ for $\chi \leqslant 0.1$ and for $R$ for $\chi \leqslant 0.2$ for all the cubic arrays.

We now turn our attention to the comparison with the approximate theories.

\section{Comparison with approximate continuum theories}

In the approximate theories, rather than solving the governing equations for the actual two-phase geometry, one solves the appropriately modified equations in a continuum with suitably defined macroscopic properties. The so-called cell theory, the self-consistent approximation, the Brinkman approximation, and Methods $A$ and $B$ recently proposed by Chang and Acrivos in a series of articles (see, for example, Refs. 9 and 10) are all examples of such theories. Of special interest to us here is the approximate theory of Brinkman ${ }^{17}$ and a recent method (equivalent to Method $A$ ) proposed by Chang and Acrivos. According to these two methods the mean flow satisfies

$$
\begin{aligned}
& \frac{d}{d x_{1}}\left(\mu\left(x_{1}\right) \frac{d u_{2}}{d x_{1}}\right)=\frac{u_{2}\left(x_{1}\right)}{\kappa\left(x_{1}\right)}, \\
& \mu\left(x_{1}\right)=1+\left(\mu^{*}-1\right) \rho\left(x_{1}\right) / \phi, \\
& 1 / \kappa\left(x_{1}\right)=\rho\left(x_{1}\right) / \phi k,
\end{aligned}
$$

subject to the conditions that $u_{2} \rightarrow 0$ as $x_{1} \rightarrow \infty$ and $\partial u_{2}$ / $\partial x_{1} \rightarrow-1$ as $x_{1} \rightarrow-\infty$. In the above expressions $u_{2}$ is the mean velocity, $\mu\left(x_{1}\right)$ and $\kappa\left(x_{1}\right)$ are, respectively, the effective viscosity and the effective permeability of the continuum (both assumed to be functions of the distance from the "interface"), $\mu^{*}$ and $k$ are the corresponding effective properties of the porous media in the bulk, i.e., as $x_{1} \rightarrow \infty$, and $\rho$ is the area fraction of the solids in the $x_{1}$ plane, the average in the bulk being $\phi$. Here for the sake of brevity we have taken the viscosity of the fluid to be unity. Clearly $\mu^{*}$ and $k$, the effective properties of the bulk, must be either supplied or estimated once again by an appropriate approximate method suitable for calculating the bulk properties. We shall presently return to the question of the proper choices for these 
two quantities, but first indicate that the main difference between the Brinkman and Chang-Acrivos approximations lies in the choice of $\rho\left(x_{1}\right)$. Whereas the latter approximation requires the actual variation in $\rho$ near the interface, the former assumes $\rho$ to take the values corresponding to the bulk phases on either side of the interface. Thus in the Brinkman approximation we have

$$
\begin{array}{ll}
\rho=\phi, & \text { for } x_{1}>0, \\
\rho=0, & \text { for } x_{1} \leqslant 0 \quad \text { (Brinkman). }
\end{array}
$$

Equations (74)-(76) can be solved analytically in the case of the Brinkman approximation, yielding

$$
\begin{aligned}
& u_{2}=-x_{1}+u_{s}, \quad \text { for } x_{1} \leqslant 0, \\
& u_{2}=u_{s} e^{-x_{1} / \sqrt{\mu^{*} k}}, \text { for } x_{1}>0, \\
& u_{s} \equiv h U_{s}=\sqrt{k / \mu^{*}}, \\
& R \equiv u_{2}\left(x_{1}+l\right) / u_{2}\left(x_{1}\right)=e^{-l / \sqrt{k \mu^{*}},},
\end{aligned}
$$

where we have chosen the ratio of $u_{2}$ at two different points separated by a distance equal to $l$ as representative of $R$. The approximation of Chang and Acrivos on the other hand requires a numerical solution to (74)-(76). A fourth-order Runge-Kutta scheme was employed to determine the slip coefficients for selected values of $\chi$.

Now we return to the question of proper choices for $k$ and $\mu^{*}$. Since $k$ for the bulk periodic arrays has been determined exactly by Zick and Homsy ${ }^{22}$ and Sangani and Acrivos, ${ }^{23}$ their reported values seem to be the most appropriate choice for $k$. These are quoted in Table II in terms of a correction factor $K$ given by $k=k_{\infty} / K$. The choice of $\mu^{*}$ on the other hand is not so straightforward as the exact calculations for this quantity are not available to date. Kim and Russel ${ }^{24}$ and Koplik, Levine, and $\mathrm{Zee}^{25}$ have addressed the problem of determining this quantity for semidilute random arrays of the spheres via the rigorous averaging methods whereas Buyevich and Shchelchkova ${ }^{26}$ have suggested an approximate continuum theory for its determination. None of these are entirely appropriate for the cubic arrays of spheres, where, indeed, even the choice of a single scalar quantity to represent a fourth-order tensor of proportionality between the stress and rate of strain tensors is itself questionable. Also, a detailed comparison of the ensemble-averaged exact numerical calculations for the permeability of the random arrays of cylinders with various approximate theories by Sangani and $\mathrm{Yao}^{8}$ has suggested that the best agreement between the approximate theories and the exact calculations for larger volume fractions is found only when the viscosity of the porous medium was taken to be either the same as that of the fluid or less than that of the fluid, which is in contrast to most self-consistent approximate schemes that predict the viscosity of the porous media to be greater than that of the fluid. In view of the above discussion it appears then that perhaps the most appropriate choice is to simply take $\mu^{*}=1$, as was done previously by Brinkman ${ }^{17}$ and Larson and Higdon. ${ }^{14,15}$

The estimates of the slip coefficient and the drag ratio obtained by using the $K$ values of Sangani and Acrivos and $\mu^{*}=1$ in Brinkman relations $(80)$ and (81) are given in Table II. We find that the agreement between the exact nu- merical calculations and Brinkman method is generally very good, particularly at lower values of $\chi$ where the Brinkman estimates coincide with the asymptotic formulas derived in Sec. III B. It is useful to note that $U_{s}$ obtained by the Brinkman method is exactly $\sqrt{k} / h$, which is the ratio of the length scales based on the macroscopic property and the microstructure of the porous medium. Thus, for instance, it is remarkable that, as $\chi$ varies from, say, 0.01 to 0.6 , Brinkman estimates of the decay rate and slip velocity remain quite accurate even though the ratio of the two length scales varies from about 7 to 0.4 . It is only for the larger volume fractions, when the regions of reverse flow begin to form near the "interface," that the Brinkman approximation begins to diverge from the exact numerical results, and, in particular, it fails to predict the reversal in the sign of $U_{s}$ at higher $\chi$. Recalling that $\chi=0.6$ corresponds approximately to $\phi=0.15$, we may expect the Brinkman theory to give reasonably accurate estimates of the slip velocity and the decay rates for the periodic as well as random arrays of spheres for $\phi<0.15$.

As mentioned earlier, the approximate method of Chang and Acrivos required solving (74)-(76) numerically. The results obtained by this method are also given in Table II where only the values of $U_{s}$ are given. The $R$ values were similar to the Brinkman approximation at low to moderate values of $\chi$ and since the decay rate becomes a rather ill-defined quantity for $\chi$ greater than approximately 0.6 , it is not very meaningful to compare the decay rates at higher values of $\chi$. (Interestingly, though, the ratio of $u_{2}$ evaluated at $x_{2}=l+L$ to that at $x_{2}=l$ did give negative values, indicating the prediction of the reverse flow by the Chang-Acrivos method and, in addition, the numerical values of the ratio were also comparable to those obtained from the exact calculations.) At very low values of $\chi$, the numerical integration of (74)-(76) must be carried out to much higher $x_{1}$ and the step size for the integration must be kept sufficiently small for resolving the presence of the particles, hence the numerical calculations for $\chi \leqslant 0.05$ may not be accurate and this is indicated by enclosing the estimates in parentheses. Apart from that, we find that the agreement between this approximate method and the exact calculations is excellent, particularly at volume fractions close to the maximum allowable for each of the three arrays. Unlike the Brinkman method, this method has been able to predict quite precisely the value of $\chi$ beyond which the slip coefficient will become negative for each of the three arrays. It must be noted that the ratio of the so-called macroscale to the microscale, i.e., $\sqrt{k} / h$, is of $O\left(\frac{1}{20}\right)$ for these arrays at close to the maximum allowable volume fractions and in spite of that the agreement between the approximate method and the exact calculations is very good.

Finally, it is of some interest to determine how sensitive the estimates obtained from the Chang-Acrivos approximation are to the choice of $\mu^{*}$. Therefore the values of $U_{s}$ for various assumed values of $\mu^{*}$ were calculated. For example, for $\chi=1$ and for the simple cubic array, the estimates of $U_{s}$ via the Chang-Acrivos approximation were found to be $0.21,0.17$, and 0.15 , respectively, for $\mu^{*}$ equal to $1.0,2.0$, and 3.0, the corresponding exact numerical result for $U_{s}$ being 0.21 . Thus we see that although the effect of varying $\mu^{*}$ is 
relatively small, any attempt at evaluating it via a separate calculation will very likely yield poor estimates of the slip coefficients.

\section{FLOW IN THE ENTRANCE REGION}

We now consider the problem of determining the velocity and pressure distributions in the entrance region of a viscous flow into a semi-infinite array of equal size spheres. Thus we shall assume that the mean flow is along the $x_{1}$ axis (cf. Fig. 1) with a magnitude $U$ and that the fluid motion satisfies the Stokes equations of motion. This problem has been examined earlier by Ishii ${ }^{16}$ who obtained the estimates of the drag forces on the spheres for the case of small volume fractions of the spheres. His result can be expressed as

$$
F^{\alpha}=6 \pi \mu U a K^{\alpha},
$$

with

$$
K^{\alpha}=1+\lambda_{1}^{\alpha} \chi+O\left(\chi^{2}\right) \quad \text { (Ishii). }
$$

For the case of a simple cubic array, Ishii reported the values of $\lambda_{1}^{\alpha}$ to be $1.44062,1.41872,1.41864$, and 1.41864 , respectively, for $\alpha=1,2,3$, and 4 . Ishii also quoted the corresponding values for the body- and face-centered cubic arrays. It was found that at such low volume fractions the flow becomes "fully developed" within approximately one or two rows of spheres and that the drag force on the first row of spheres was slightly greater than the rest in the case of a simple cubic array, but slightly lower than the rest in the case of the body- and face-centered cubic arrays for which the second row of spheres experienced the greatest drag force. This slight difference in the behavior of the simple vis-a-vis body- and face-centered cubic arrays was attributed to the presence of a flow meandering between the first two rows of spheres, which are arranged in a staggered configuration in body- and face-centered cubic arrays.

We follow the method described in Sec. III A to obtain a complete formal solution to the fluid motion for the present case:

$$
\begin{aligned}
& u_{1}=U+\sum_{\alpha}\left[\mathbf{G}^{\alpha}\left(\Psi_{1}-\frac{\partial^{2} \Psi_{2}}{\partial x_{1}^{2}}\right)+\mathbf{H}^{\alpha}\left(\frac{\partial \Psi_{1}^{*}}{\partial x_{1}}\right)\right. \\
&\left.+\mathbf{L}^{\alpha}\left(-\frac{3}{4} \frac{\partial^{3} \Psi_{1}^{*}}{\partial x_{1}^{3}}-2 \Delta_{4} \frac{\partial \Psi_{2}}{\partial x_{1}}\right)\right] \\
& u_{2}=\sum_{\alpha}\left[\mathbf{G}^{\alpha}\left(-\frac{\partial^{2} \Psi_{2}}{\partial x_{1} \partial x_{2}}\right)+\mathbf{H}^{\alpha}\left(\frac{\partial \Psi_{1}}{\partial x_{2}}\right)\right. \\
&\left.+\mathbf{L}^{\alpha}\left(\frac{\partial^{3} \Psi_{1}}{\partial x_{2}^{3}}-2 \Delta_{4} \frac{\partial \Psi_{2}}{\partial x_{2}}\right)\right] \\
& u_{3}=\sum_{\alpha}\left[\mathbf{G}^{\alpha}\left(-\frac{\partial^{2} \Psi_{2}}{\partial x_{1} \partial x_{3}}\right)+\mathbf{H}^{\alpha}\left(\frac{\partial \Psi_{1}}{\partial x_{3}}\right)\right. \\
&\left.+\mathbf{L}^{\alpha}\left(\frac{\partial \Psi_{1}}{\partial x_{3}^{3}}-2 \Delta_{4} \frac{\partial \Psi_{2}}{\partial x_{3}}\right)\right], \\
& \frac{\partial P}{\mu} \frac{\sum_{\alpha}}{\partial x_{i}}\left[\mathbf{G}^{\alpha}\left(\frac{4 \pi}{\tau} \delta_{i 1} \delta\left(x_{1}-x_{1}^{\alpha}\right)-\frac{\partial^{2} \Psi_{1}}{\partial x_{1} \partial x_{i}}\right)\right. \\
&\left.\quad-2 \mathbf{L}^{\alpha} \Delta_{4} \frac{\partial \Psi_{1}}{\partial x_{i}}\right]
\end{aligned}
$$

TABLE III. The numerical results for drag on the finite number of arrays for the case of mean flow perpendicular to the arrays. $N_{t}$ : the number of arrays, SQ: square; SC: simple cubic; and BCC: body-centered cubic.

\begin{tabular}{cccccc}
\hline \hline Array & $\chi$ & \multicolumn{1}{c}{$K^{1}$} & $K^{2}$ & $K^{3}$ & $N_{\iota}$ \\
\hline \multirow{2}{*}{ SQ } & 1.0 & 43.9 & $\cdots$ & $\cdots$ & 1 \\
& 0.9 & 20.19 & $\cdots$ & $\cdots$ & 1 \\
& & & & & \\
SC & 1.0 & 42.8 & 44.5 & $\cdots$ & 3 \\
& 0.9 & 19.68 & 19.17 & $\cdots$ & 3 \\
& & & & & \\
& 1.0 & 99.7 & $\ldots$ & $\ldots$ & 2 \\
BCC & 1.0 & 96.8 & 168.7 & $\cdots$ & 3 \\
& 1.0 & 95.9 & 167.3 & $\cdots$ & 4 \\
& 1.0 & 96.1 & 166.6 & 165.1 & 5 \\
& 1.0 & 98.0 & 165.9 & 162.6 & 6 \\
\hline \hline
\end{tabular}

where the differential operators $\mathbf{G}^{\alpha}, \mathbf{H}^{\alpha}$, and $\mathbf{L}^{\alpha}$ are defined in the same manner as in Sec. III A except that the constants $B_{00}^{\alpha}$ and $A_{n m}^{\alpha}, B_{n m}^{\alpha}$, and $C_{n m}^{\alpha}$ are zero for all $m$ not exactly divisible by 4 . The above solution at first may suggest that $\partial P / \partial x_{1}$ or $u_{1}$ is singular at points lying in the planes $x_{1}=x_{1}^{\alpha}$. However, it must be noted that this is not the case as $\Psi_{1}-\partial^{2} \Psi_{2} / \partial x_{1}^{2}$ is equal to $\left(\partial^{2} / \partial x_{2}^{2}+\partial^{2} / \partial x_{3}^{2}\right) \Psi_{2}$, which is clearly a regular function. The terms containing $\mathbf{H}^{\alpha}$ result upon taking the Laplacian of the terms associated with $\mathbf{G}^{\alpha}$ and hence they are also regular at all points in the fluid. Since $\partial \Psi_{1} / \partial x_{1}$ is, however, singular on the planes $x=x_{1}^{\alpha}$, this singularity must be removed in calculating the terms associated with $\mathrm{H}^{\alpha}$ in $u_{1}$; this is indicated by an asterisk on $\Psi_{1}$ in the expression for $u_{1}$. The same arguments apply to the $\Psi_{1}$ term operated by $L$ in $u_{1}$. Finally, it is interesting to note that the expressions for the velocity and pressure derived here resemble significantly those given earlier by Sangani and Acrivos ${ }^{23}$ (who employed the triply periodic singular solutions of $\mathrm{Ha}-$ simoto $^{21}$ ) even though the nature of higher-order tensors, which relate the strengths of singularities to the mean flow vector [by relations similar to (37)], having the cubic symmetry in the case of the problem examined by Sangani and Acrivos is quite different from those depending upon the orientation of the semi-infinite arrays considered in the present study. This apparent similarity between the two expressions derived by two different methods is partially due to the fact that (as explained in Ref. 27) the cubic symmetry was not fully exploited in the solution of Sangani and Acrivos.

The above expressions for the velocity and pressure were suitably truncated and the resulting equations were solved numerically. We first present the results for $K^{a}$ for the case of a finite number $N_{t}$ of planar arrays. Because of the symmetry about the midplane, we have given the values of $K^{\alpha}$ for only one-half the number of arrays in Table III. Since the maximum deviation in the drag on the spheres in different planes is seen at $\chi=1$, only the results for larger $\chi$ are presented. The results for $N_{t}=1$ correspond to the case of a single planar array. We find that the drag force on a single planar square array is surprisingly close to that for an infinite simple cubic array as determined by Zick and Homsy ${ }^{22}$ and Sangani and Acrivos. ${ }^{23}$ Thus, for example, the $K$ values at 
$\chi=0.9$ and 1.0 for the square planar arrays are 20.2 and 43.9 compared with the corresponding values of 20.1 and 42.4 for the simple cubic array as determined by Sangani and Acrivos. Thus we conclude that the effect of the presence of another row of spheres at a distance greater than $2 h$ is very small as long as this second row of spheres is aligned exactly behind the first and consequently the entrance flow region for the simple cubic arrays is very small.

The results for the body-centered cubic arrangement show that, unlike the case of a simple cubic array, the drag force on the first row of spheres is much smaller than that on the second or third row of spheres. Calculations with a larger $N_{t}$ showed that for this array, the drag force on the second row of spheres is the greatest although the difference among the subsequent rows is indeed very small. Thus the numerical results confirm the findings of Ishii who, as mentioned earlier, considered the case of very small volume fractions of the spheres. We also found that the results for the finite number of planar arrays in a face-centered cubic arrangement are qualitatively similar to those of a body-centered cubic arrangement.

In view of our observations with a finite number of planar arrays, it seems that the situation of the semi-infinite array can be simulated simply by considering a case of finite $N_{t}$ of, say, $O(10)$. The results of numerical computations with $N_{t}=11$ (except for a few cases at higher $\chi$ for which $N_{t}$ $=13$ was chosen) for various arrays are given in Table IV where the drag forces on the four outermost rows of spheres are given owing to space consideration. The corresponding $K$ values for the infinite cubic arrays are reported in Table II. As in the case of the shear flow we may define a pressure slip coefficient in the following manner. For larger $x_{1}$ we expect the flow to become "fully developed" whereby the pressure, suitably averaged, will decrease linearly with $x_{1}$. Thus we expect

$$
\left\langle P\left(x_{1}\right)\right\rangle-P_{-\infty}=-\mu U\left(x_{1}-h P_{s}\right) / k, \quad x_{1} \gg h,
$$

where $\boldsymbol{P}_{-\infty}$ is the pressure in the fluid far upstream of the array and $k$ is the permeability of the bulk as defined in Sec. III. For the sake of definiteness, we shall evaluate the average pressure by taking an average over a two-dimensional

TABLE IV. The results for the drag forces and the pressure slips in semiinfinite arrays.

\begin{tabular}{ccccccc}
\hline \hline Array & $\chi$ & $K^{\prime}$ & $K^{2}$ & $K^{3}$ & $K^{4}$ & $P_{*}$ \\
\hline & 0.3 & 1.71 & 1.84 & 1.83 & 1.83 & 0.072 \\
BCC & 0.5 & 2.92 & 3.41 & 3.39 & 3.39 & 0.152 \\
& 0.9 & 27.6 & 43.7 & 42.8 & 42.8 & 0.388 \\
& 1.0 & 97.2 & 167 & 164 & 162 & 0.42 \\
& & & & & & \\
& 0.5 & 3.27 & 3.58 & 3.57 & 3.57 & 0.119 \\
FCC & 0.9 & 42.8 & 65.2 & 65.1 & 65.1 & 0.48 \\
& 1.0 & 273 & 417 & 420 & 414 & 0.59 \\
& & & & & & \\
& 0.5 & 2.90 & 2.84 & 2.84 & 2.84 & -0.04 \\
SC & 1.0 & 42.7 & 43.8 & 42.5 & 42.4 & -0.08 \\
\hline \hline
\end{tabular}

unit cell situated at a distance $l / 2$ from the center of an $m$ th row of spheres, where $m$ is a sufficiently large integer to ensure that the flow is "fully developed" there. With this definition of the pressure slip, and using an overall momentum balance that relates the pressure drop to the drag forces on the spheres, it can be shown that

$$
P_{s}=l \sum_{\alpha=1}^{\alpha=m} \frac{\left(K^{\infty}-K^{\alpha}\right)}{K^{\infty} h}, m \gg 1 .
$$

In practice, since the flow becomes "fully developed" very quickly, $m=6$ is sufficient. Thus with $N_{t}=11$, the $K$ value of the sixth row was used as $K^{\infty}$ and $P_{s}$ thus computed are also given in Table IV. We find that for the simple cubic array this quantity is at most $O(0.1)$ and for the body- and face-centered arrays it is $O(0.5)$, all of which are quite small and suggest that the entrance lengths for the periodic arrays are less than one-half the unit cell width. Finally, it may be noted that at very low $\mathcal{X}$, the asymptotic analysis of Ishii ${ }^{16}$ gives $P_{s}=\lambda_{s} \chi$ with $\lambda_{s}$ equal to $-0.0441,0.1589$, and 0.1091 , respectively, for the simple, body-centered and facecentered cubic arrays. Our numerical calculations suggest that these estimates are good for $\chi$ less than 0.1 .

We now discuss the approximate continuum theories. Here, the mean flow is constant and the pressure gradient is nonzero. Therefore according to the continuum theories we have

$$
-\frac{d P}{d x}=\frac{\rho\left(x_{1}\right) U}{k \phi},
$$

where we have taken $\mu$ and $\mu^{*}$ to be unity and $k, \rho$, and $\phi$ have the same meaning as in Sec. III. It can be shown that an integration of the above equation from $x_{1}=-\infty$ to $x_{1}^{m}$ $+l / 2(m \gg 1)$ yields

$$
P_{s} \equiv 0 \quad \text { (approximate theories) }
$$

for both the Brinkman and Chang-Acrivos theories. This result may be also explained as follows. In the case of a periodic array, $\rho\left(x_{1}\right)$ has the same distribution near $x_{1}=0$ for positive $x_{1}$ as in the bulk of the array, and hence there is no entrance region effect. Thus both theories fail to give a nonzero estimate of the pressure slip. The reason for this failure obviously lies in not accounting for the fact that although $\rho\left(x_{1}\right)$ near $x_{1}=0$ varies in the same manner as in the bulk for the periodic arrays, the permeability in the entrance region can be different than in the bulk. Fortunately, our exact calculations suggest that the entrance region is very small for the complete range of volume fractions and hence the actual numerical values of the pressure slip are indeed small. Thus the failure of the approximate theories should be regarded as minor.

\section{THERMAL CONDUCTION}

In this section we shall consider the problem of determining the temperature distribution in the entrance region of the heat flux by conduction into a semi-infinite array. The formal solution for this problem can be obtained in terms of the derivatives of $\Psi_{1}$, which is the fundamental singular solution of the Laplace equation. Unlike the problems considered in the Secs. III and IV, however, the solution for a semiinfinite array contains a constant that is infinitely large and 
thus has a slightly undesirable feature. To avoid this, we first start with a solution for a finite row, $N_{t}$, of spheres, and since our primary interest here is in determining a quantity $T_{s}$ [defined via (97)] related to the entrance effect, we calculate it first for a finite $N_{t}$ and then take the limit as $N_{t} \rightarrow \infty$. Thus we obtain

$$
\begin{aligned}
T= & x_{1}+\mathscr{C}_{N_{t}}+\sum_{\alpha=1}^{\alpha=N_{t}} \sum_{n=1}^{n=\infty} \sum_{m=0}^{m \leq n / 4} A_{n m}^{\alpha} \Delta_{4 m} \frac{\partial^{n-4 m}}{\partial x_{1}^{n-4 m}} \\
& \times\left(\Psi_{1}-\frac{2 \pi}{\tau}\left|x_{1}-x_{1}^{\alpha}\right|\right),
\end{aligned}
$$

where it is assumed that the temperature gradient far away from the array is unity. It is easy to verify that the above solution is regular at all points outside the spheres. Now we shall require that $T=x_{1}$ as $x_{1} \rightarrow-\infty$ so that

$$
\mathscr{C}_{N_{t}}=\frac{4 \pi}{\tau} \sum_{\alpha=1}^{\alpha=N_{t}} A_{10}^{\alpha} .
$$

Since $A_{10}^{\alpha}$ is expected to become a constant for larger $\alpha$, the constant $\mathscr{C}_{N}$, would have been infinite if we had started with $N_{\imath}=\infty$. Now for $1 \ll m \ll N_{t}$, we expect the temperature field to be "fully developed" near $x_{1}^{m}$ and we may evaluate the average temperature gradient in the bulk by

$$
\begin{aligned}
\frac{d\langle T\rangle}{d x_{1}} & =\frac{\left(\langle T\rangle_{x_{1}^{m}+l / 2}-\langle T\rangle_{x_{1}^{m}-l / 2}\right)}{l} \\
& =1-8 \pi A_{10}^{m} / \tau l,
\end{aligned}
$$

which is independent of $\mathscr{C}_{N_{t}}$. Let us assume, with no loss of generality, that the conductivity of the suspending medium is unity and that of the spheres is $\beta$. Since the net heat flux through the array is unity, the overall conductivity of the bulk $D^{*}$ is simply the inverse of the mean temperature gradient calculated above, i.e.,

$$
D^{*}=1 /\left(1-8 \pi A_{10}^{m} / \tau l\right) \text {. }
$$

Now we may define the temperature $\operatorname{slip} T_{s}$ by the following expressions:

$$
\begin{aligned}
& T=x_{1}+\text { e.d.t. as } x_{1} \rightarrow-\infty, \\
& \langle T\rangle_{x_{1}^{m}+l / 2}=D^{*-1}\left(x_{1}^{m}+l / 2+h T_{s}\right), \quad m \gg 1 .
\end{aligned}
$$

The above definition of the temperature slip can be shown to lead to the following formula:

$$
h T_{s}=8 \pi D^{*} \sum_{\alpha=1}^{\alpha=m} \frac{\left(A_{10}^{m}-A_{10}^{\alpha}\right)}{\tau} .
$$

As in Secs. III and IV, the formal solution for the temperature field was suitably truncated and the resulting equations were solved numerically for selected values of $\chi$ and $\beta$. It was found that $T_{s}$ approached nearly a constant value for $N_{t}>9$ and $m=5$ in almost all the cases considered. The results are presented in Table V. It should be noted that $A_{10}$

\begin{tabular}{|c|c|c|c|c|c|c|}
\hline$B$ & $D^{*}$ & $T_{s}$ & $A_{10}^{X}=$ & $A_{10}^{2}$ & $A_{10}^{3}$ & Array \\
\hline 50 & (9.7) & $(-1.4)$ & $(0.25)$ & $(0.22)$ & $(0.22)$ & \multirow{7}{*}{$\mathrm{BCC}$} \\
\hline 30 & 8.1 & -1.2 & 0.25 & 0.21 & 0.21 & \\
\hline 20 & 6.77 & -0.9 & 0.24 & 0.21 & 0.21 & \\
\hline 10 & 4.67 & -0.55 & 0.22 & 0.19 & 0.19 & \\
\hline 5 & 3.03 & -0.26 & 0.18 & 0.16 & 0.16 & \\
\hline 0.3 & 0.48 & -0.07 & -0.23 & -0.26 & -0.26 & \\
\hline 0.0 & 0.22 & -0.23 & -0.64 & -0.89 & -0.89 & \\
\hline 50 & (6.5) & $(0.7)$ & $(0.25)$ & $(0.27)$ & $(0.27)$ & \multirow{4}{*}{ SC } \\
\hline 30 & 5.52 & 0.55 & 0.24 & 0.26 & 0.26 & \\
\hline 20 & 4.74 & 0.42 & 0.24 & 0.25 & 0.25 & \\
\hline 0 & 0.34 & 0.025 & -0.62 & -0.61 & -0.61 & \\
\hline 20 & 8.34 & -0.65 & 0.21 & 0.20 & 0.20 & \multirow[b]{2}{*}{ FCC } \\
\hline 10 & 5.45 & -0.39 & 0.20 & 0.18 & 0.18 & \\
\hline \multicolumn{7}{|c|}{$\beta=\infty$} \\
\hline$\chi$ & $D^{*}$ & $T_{s}$ & $A_{\text {io }}^{i}$ & $A_{10}^{2}$ & $A_{10}^{3}$ & Array \\
\hline 0.95 & 5.82 & -0.82 & 0.24 & 0.20 & 0.20 & \multirow{3}{*}{$\mathrm{BCC}$} \\
\hline 0.90 & 4.10 & -0.49 & 0.21 & 0.18 & 0.19 & \\
\hline 0.50 & 1.28 & -0.01 & 0.055 & 0.053 & 0.053 & \\
\hline
\end{tabular}
represents the magnitude of the thermal dipole and may be taken as a representative quantity for determining when the temperature field has become "fully developed." As one might expect, the spheres in the first row are less "polarized" compared to the rest in the case of a simple cubic array and hence the dipole strength monotonically increases with $\alpha$. As in the case of flow, we see once again that the qualitative
TABLE V. The thermal dipoles and the temperature slip coefficients.

behavior of the simple cubic array is different from that of the body- and face-centered cubic arrays. We also note that although $T_{s}$ becomes zero for $\beta=1$, it does not reverse its sign as $\beta$ is decreased further. Finally, since the bulk conductivity becomes infinite as $\beta \rightarrow \infty$ and $\chi \rightarrow 1$, the numerical calculations become difficult and the results for higher $\chi$ and $\beta$ may not have converged; this is indicated by enclosing the particular values in parentheses.

Now once again we discuss the predictions of the approximate theories. Since the heat flux is taken to be unity, the gradient of the mean temperature in the medium is given by

$$
\frac{d\langle T\rangle}{d x_{1}}=\frac{1}{D\left(x_{1}\right)},
$$

where $D\left(x_{1}\right)$ is the effective conductivity of the medium, which is usually taken as

$$
D\left(x_{1}\right)=1+\left(D^{*}-1\right) \rho\left(x_{1}\right) / \phi .
$$

Now (99) can be integrated to determine the temperature slip coefficient for both the Brinkman and Chang-Acrivos approximations. Clearly the former gives the value of the slip to be identically zero whereas the latter does not even correctly predict the average temperature gradient in the medium since the average of $1 / D\left(x_{1}\right)$ over a three-dimensional unit cell is not equal to $1 / D^{*}$. Therefore we must modify (100) slightly and take instead

$$
\frac{1}{D\left(x_{1}\right)}=1+\frac{\left(1 / D^{*}-1\right) \rho\left(x_{1}\right)}{\phi} \text {. }
$$

Of course, at small volume fractions of the spheres or for $\beta$ close to unity, both (100) and (101) are equivalent to the leading order in $D^{*}-1$ and hence the use of either of the expressions in constructing approximations is equally justi- 
fied. Now, substituting (101) into (99) we obtain the correct behavior of $\langle T\rangle$ for $x_{1}>0$ and, in particular, we find that in this case, also, $T_{s} \equiv 0$. Thus once again the approximate theories have predicted the slip coefficient to be zero while the exact calculations have given small but nonzero values.

We end this section by noting that recently Chang and Acrivo ${ }^{13}$ have considered a similar problem in which a random suspension of spheres is in contact with a wall maintained at a constant temperature at $x_{1}=0$ and estimated a quantity similar to $T_{s}$. Once again this quantity is identically zero for the case of periodic array provided that the wall is exactly $l / 2$ distance away from the wall and if we calculate the temperature slip (or offset as referred to by Chang and Acrivos) in the manner defined here. Once again the numerical method presented in this paper can be applied to the case examined by Chang and Acrivos and we expect that the exact calculations will yield rather small numerical values for this quantity.

\section{DIFFUSION ACCOMPANIED BY A CHEMICAL REACTION}

\section{A. Statement of the problem and numerical results}

As a last application of the planar singular solutions we consider a problem often encountered in the modeling of a reaction in porous catalysts or adsorption columns where it is a common practice to treat the two-phase medium as an effective continuum whose diffusivity is assumed to be a function of the volume fraction of the solid phase and the diffusivities of the individual phases but not that of the reaction rate. Such an assumption appears reasonable if the reaction is sufficiently slow. On the other hand, it is easy to see that replacing the two-phase medium by an effective continuum may not be satisfactory if the reaction is fast or that, even if the two-phase medium can be treated as an effective continuum for the case of moderate reaction rates, the overall diffusivity may be dependent on the rate constant of the reaction. Thus it is of interest to determine the bulk diffusivity of a two-phase medium in the presence of a moderately fast chemical reaction. Unlike the formulation of the problems for determining the overall conductivity or Darcy permeability where one assumes that a unit temperature or pressure gradient is imposed throughout the medium and then determines the average flux through the two-phase medium, the formulation of the problem of predicting the bulk diffusivity of a reacting medium seems to be more difficult as the presence of a chemical reaction, which acts as a sink for the diffusing substance, also requires the presence of a source term throughout the medium to maintain a constant concentration gradient in the medium. Such formulations have been considered by several previous investigators and the reader is referred to the work of Felderhof ${ }^{28,29}$ for the references to these efforts. Using one of these formulations, Felderhof has determined what he referred to as the rate constants of the diffusion-controlled chemical reactions for the cases of a random array having small volume fractions of the spheres and for spatially periodic arrays of spheres with arbitrary volume fractions. Since our primary interest in this paper is to assess the effective continuum theories, we shall consider first relatively slow reactions and compare the re- sults obtained by the exact numerical analysis with those obtained via the approximate theories of Brinkman and of Chang and Acrivos. We find that the Brinkman theory provides better estimates in this case. Next we consider moderately fast reactions and, assuming that the two-phase medium can be regarded as a Brinkman continuum, we determine the effective diffusivity of the reacting medium by matching the decay rates of the mean concentration in the actual twophase medium to that in the Brinkman continuum. The estimates of the diffusivities thus obtained show that the bulk diffusivities of the reacting media are greater than that of the nonreacting media. This observation is further supported by an asymptotic analysis for small $\chi$ and slow reactions.

Let us assume that the spherical particles in the previous sections are now the sites of a first-order chemical reaction and that the diffusivity of the reactant is $\beta$ inside the particles and unity outside the particles. We shall assume that the concentrations are sufficiently small so that the fluxes are given by Fick's law. Thus we have

$$
\begin{aligned}
& \nabla^{2} c=0, \quad \text { in the continuous phase, } \\
& \nabla^{2} c=\mathscr{R} c / \beta h^{2}, \quad \text { inside the spheres, }
\end{aligned}
$$

where $c$ is the concentration of the reactant and $\mathscr{R} / h^{2}$ is the rate constant of the reaction. The boundary conditions are that $c \rightarrow 0$ as $x_{1} \rightarrow \infty, c$ is continuous at the surface of the particles and periodic in the $x_{1}$ planes, and

$$
\begin{aligned}
& \beta(\nabla c \cdot n)_{-}=(\nabla c \cdot n)_{+}, \quad \text { on the spheres, } \\
& c=-x_{1}+h C_{s} \text { as } x_{1} \rightarrow-\infty,
\end{aligned}
$$

where the first equation is the continuity of the normal flux at the surface of the spheres, $n$ is the unit normal at the surface of the spheres, and the second equation defines the concentration slip, a quantity that we wish to determine.

In certain situations it is more appropriate to assume that the reaction is occurring only at the surface of the spheres and not inside the spheres. In this case we have, in lieu of (103) and (104), the following relations:

$$
\begin{aligned}
& \nabla c \cdot n=\mathscr{R}_{s} c / h, \quad \text { on the spheres, } \\
& c=\text { const, inside the spheres, }
\end{aligned}
$$

where $\mathscr{R}_{s} / h$ is the rate constant for the surface reaction. We note that the case of reaction inside the particles reduces to that on the surface if we take $\beta=\infty$ and $\mathscr{R}=3 \mathscr{R}_{s} / \chi$.

A formal solution for $c$ for any point in the suspending medium can be written at once in terms of the function $\Psi_{1}$ :

$$
c(\mathbf{r})=c^{*}\left(x_{1}\right)+\sum_{\alpha} \mathbf{G}^{\alpha} \Psi_{1}\left(\mathbf{r}-\mathbf{r}^{\alpha}\right),
$$

with

$$
c^{*}\left(x_{1}\right)=-\frac{x_{1}}{2}-\frac{2 \pi}{\tau} \sum_{\alpha} \mathbf{G}^{\alpha}\left|x_{1}-x_{1}^{\alpha}\right|,
$$

where $\mathbf{G}^{\alpha}$ is the differential operator as before [cf. (41)] with $A_{n m}^{\alpha}$ equal to zero for all $m$ not divisible by 4 . It is easy to verify that the above solution satisfies the boundary conditions at infinity and that

$$
\frac{\tau}{8 \pi}=-\sum_{\alpha} A_{\infty}^{\alpha}
$$




$$
C_{s}=8 \pi \sum_{\alpha} \frac{\left(A_{10}^{\alpha}-x_{1}^{\alpha} A_{00}^{\alpha}\right)}{h \tau} .
$$

Now to determine $A_{n m}^{\alpha}$, we expand $c$ near the center of each sphere in spherical harmonics. Let the expansion near the center of a sphere on the sth row be written as

$$
c^{s}=\sum_{n, m}\left(D_{n m}^{s}+E_{n m}^{s} r^{-2 n-1}\right) Y_{n}^{m},
$$

where $r$ is measured from the center of the sphere. Here $E_{n m}^{s}$ is related to $A_{n m}^{s}$ simply by

$$
E_{n m}^{s}=(-1)^{n-m}(n+m) !(n-m) ! A_{n m}^{s} 2^{1-m},
$$

since this singular term results upon differentiating $1 / r$ in $\Psi_{1}$ whereas the constant $D_{n m}^{s}$ that results from the regular part of $c$ at $\boldsymbol{r}^{s}$ is obtained by applying (54). Thus

$D_{n m}^{s}=\epsilon_{m}\left(\frac{\partial}{\partial x_{1}}\right)^{n-m} \Delta_{m}\left(c-\mathbf{G}^{s} \frac{1}{\left|\mathbf{r}-\mathbf{r}^{s}\right|}\right)_{\mathbf{r}=\mathbf{r}^{s}}$.

Now, inside a sphere located on the sth row, $c$ can be expressed in series of modified spherical Bessel functions and upon applying the boundary conditions of the continuity of $c$ and the normal flux at the surface of the sphere [cf. (104)], we obtain

$$
E_{n m}^{s}=L_{n} D_{n m}^{s} a^{2 n+1},
$$

where

$$
L_{n}=\frac{n i_{n}(z)-\beta z i_{n}^{\prime}(z)}{(n+1) i_{n}(z)+\beta z i_{n}^{\prime}(z)}, \quad z^{2} \equiv \frac{\mathscr{R} \chi^{2}}{\beta} .
$$

Here $i_{n}$ is a modified spherical Bessel function and $i_{n}^{\prime}$ is its derivative. We note that for the special case of the surface reaction mentioned earlier we have

$$
\begin{aligned}
& L_{0}=-\mathscr{R}_{s} \mathcal{X} /\left(1+\mathscr{R}_{s} \mathcal{X}\right), \\
& L_{n}=-1, \text { for } n \geqslant 1 \quad(\beta=\infty) .
\end{aligned}
$$

Now the formal solution can be suitably truncated and the resulting unknowns $A_{n m}^{\alpha}$ can be determined numerically for the selected values of $\chi, \mathscr{R}$, and $\beta$ by making use of the above relations. The numerical results are presented in Table VI. We see that $C_{s}$ approaches infinity as $\chi$ or $\mathscr{R} \rightarrow 0$. This was, of course, expected since the spheres are the only sources of sinks and if their volume decreases then it must be compensated by higher reaction rates or higher $c$ in order that the steady influx of the diffusing species is consumed. However, before we discuss the numerical results further, it proves useful to describe approximate continuum theories for the present situation. Here, the presence of sinks within the spheres must appear as a sink term smeared through the continuum and the average concentration therefore satisfies

$$
\frac{d}{d x_{1}}\left(D_{R}\left(x_{1}\right) \frac{d c\left(x_{1}\right)}{d x_{1}}\right)=\frac{\rho\left(x_{1}\right) \mathscr{R} c\left(x_{1}\right)}{h^{2}},
$$

with

$$
D_{R}\left(x_{1}\right)=1+\frac{\left(D_{R}^{*}-1\right) \rho\left(x_{1}\right)}{\phi} .
$$

\begin{tabular}{|c|c|c|c|c|c|c|c|}
\hline \multicolumn{8}{|c|}{$\beta=1$} \\
\hline \multirow[b]{2}{*}{$x$} & \multirow[b]{2}{*}{$D^{*}$} & \multirow[b]{2}{*}{$\mathscr{H}$} & \multicolumn{2}{|c|}{ Exact } & \multirow{2}{*}{$\begin{array}{l}\mathrm{Br} \\
C_{\mathrm{s}}\end{array}$} & \multirow{2}{*}{$\begin{array}{l}\text { CA } \\
C_{s}\end{array}$} & \multirow[b]{2}{*}{$D_{R}^{*}$} \\
\hline & & & $C_{s}$ & $S$ & & & \\
\hline \multirow[t]{3}{*}{0.3} & 1.0 & 0.2 & 16.5 & 0.933 & 16.5 & 11.6 & 1.004 \\
\hline & & 0.4 & 11.7 & 0.906 & 11.7 & 9.9 & 1.009 \\
\hline & & 1.0 & 7.47 & 0.857 & 7.4 & 7.1 & 1.022 \\
\hline \multirow[t]{3}{*}{0.5} & 1.0 & 1.0 & 3.51 & 0.719 & 3.4 & 3.4 & 1.038 \\
\hline & & 2.0 & 2.54 & 0.632 & 2.4 & 2.4 & 1.077 \\
\hline & & 4.0 & 1.88 & 0.534 & 1.7 & 1.7 & 1.152 \\
\hline \multirow[t]{2}{*}{0.8} & 1.0 & 1.0 & 1.72 & 0.511 & 1.7 & 1.7 & 1.031 \\
\hline & & 4.0 & 0.90 & 0.28 & 0.85 & 0.90 & 1.120 \\
\hline \multirow[t]{3}{*}{0.9} & 1.0 & 4.0 & 0.70 & 0.21 & 0.71 & 0.78 & 1.079 \\
\hline & & 8.0 & 0.50 & 0.12 & 0.50 & 0.57 & 1.154 \\
\hline & & 16 & 0.36 & 0.05 & 0.36 & 0.41 & 1.276 \\
\hline \multicolumn{8}{|c|}{$\beta=\infty$} \\
\hline \multirow[b]{2}{*}{$\chi$} & & & \multicolumn{2}{|c|}{ Exact } & $\mathrm{Br}$ & $\mathrm{CA}$ & \\
\hline & $D^{*}$ & $\mathscr{R}_{\mathrm{s}}$ & $C_{s}$ & $\boldsymbol{S}$ & $C_{s}$ & $C_{\mathrm{s}}$ & $D_{R}^{*}$ \\
\hline \multirow[t]{4}{*}{0.5} & 1.29 & 0.1 & 3.95 & 0.796 & 3.91 & 3.3 & 1.29 \\
\hline & & 0.6 & 1.70 & 0.583 & 1.60 & 1.36 & 1.40 \\
\hline & & 1.0 & 1.37 & 0.508 & 1.24 & 1.07 & 1.48 \\
\hline & & 2.0 & 1.06 & 0.406 & 0.87 & 0.79 & 1.67 \\
\hline \multirow[t]{3}{*}{0.8} & 2.62 & 0.3 & 0.93 & 0.644 & 0.98 & 1.25 & 2.69 \\
\hline & & 2.0 & 0.36 & 0.348 & 0.38 & 0.54 & 3.12 \\
\hline & & 4.0 & 0.27 & 0.248 & 0.27 & 0.40 & 3.58 \\
\hline \multirow[t]{3}{*}{0.9} & 4.1 & 0.6 & 0.39 & 0.57 & 0.50 & 1.0 & 4.28 \\
\hline & & 2.0 & 0.18 & 0.38 & 0.27 & 0.60 & 4.69 \\
\hline & & 4.0 & 0.12 & 0.27 & 0.19 & 0.46 & 5.27 \\
\hline
\end{tabular}

Here $D_{R}^{*}$ is the bulk or overall diffusivity of the reacting medium and $D_{R}\left(x_{1}\right)$ is the variable diffusivity of the continuum. Of course, in the case of the Brinkman approximation,
TABLE VI. Diffusion accompanied by a chemical reaction in a semi-infinite body-centered cubic array. Brinkman (Br) and Chang-Acrivos (CA) approximations are calculated by choosing $D_{R}^{*}=D^{*}$.

for which $\rho=\phi$ for $x_{1}>0$, the two diffusivities are equal to each other and the integration of (118) can be carried out analytically yielding

$$
C_{s}=1 / \sqrt{\phi D_{R}^{*} \mathscr{R}} \quad \text { (Brinkman) }
$$

and

$$
S \equiv c\left(x_{1}+l\right) / c\left(x_{1}\right)=e^{-l \sqrt{\phi: x^{2} / h^{2} D_{k}^{*}}},
$$

where $S$ is a measure of the decay rate of the concentration in the medium. These simple expressions already suggest the expected behavior at small $\phi$ or $\chi$ and for slow reactions. For a more precise comparison with the numerical results, however, we need to specify the bulk diffusivity of the reacting medium. As mentioned in the introduction of this section, a common practice is to simply choose the bulk diffusivity to be independent of the rate constant and to be a function of $\chi$ and $\beta$ only. We expect this to be a reasonable assumption for slow reactions. The estimates of the bulk diffusivity in the absence of a chemical reaction have been reported in the 
literature and in fact they are the same as $D^{*}$ presented in Sec. $V$ because of the identical nature of the governing equations for the problems of determining the overall conductivity and bulk diffusivity. Thus we have taken $D_{R}^{*}=D^{*}$ in (119) and integrated the resulting equations numerically by a fourth-order Runge-Kutta scheme to obtain the estimates of $C_{s}$ via the Chang-Acrivos approximations; the estimates from the Brinkman approximation can be, of course, directly evaluated from (120) and (121) by substituting $D_{R}^{*}$ $=D^{*}$. The results of these calculations are compared with the exact numerical results in Table VI where, because of the space limitations, we have presented results only for the body-centered cubic arrays with $\beta=\infty$ (the surface reaction case) and $\beta=1$. We find that the Brinkman theory gives surprisingly good estimates of $C_{s}$ for nearly the complete range of $\chi$ even for the moderately fast reactions. We note that $\sqrt{\mathscr{R}}$ or $\mathscr{R}_{s}$ may be regarded as the ratio of the microscale to the reaction length scale. Thus it is quite remarkable that the Brinkman approximation continues to give fairly accurate estimates of $C_{s}$ even when the ratio of these scales is 4 . Unlike the case of shear flow examined in Sec. III, the approximate theory of Chang and Acrivos gives inferior estimates of $C_{s}$.

Our numerical calculations showed that $S^{\alpha}$ became nearly constant to at least three significant digits for $\alpha$ greater than 3 in almost all the cases considered in Table VI. (Depending upon the decay rate, the number of rows $N_{t}$ was chosen in the range 20-100.) Therefore it seems appropriate to treat the two-phase medium as an effective continuum for, say, $\alpha>3$ and to define the bulk diffusivity of this continuum by matching $S$ obtained from the exact calculations (with $\alpha=4$ ) with that given by Brinkman theory [cf. (121)]. The estimates of the bulk diffusivity thus obtained are also given in Table VI. In the limit of very slow reactions the estimates of $D_{R}^{*}$ thus obtained converged to $D^{*}$. For faster reactions the bulk diffusivity of the reacting medium is found to be greater than that of a nonreacting medium. This is also supported by the asymptotic analysis to be presented in Sec. VI B. We wish to note that since our formulation of the effective property of the reacting media is quite different from that employed by Felderhof or other investigators, we have not compared our results with the previous investigators.

To be sure, we must also inquire how the estimates of $C_{s}$ obtained from Brinkman theory compare with those from the exact calculations if we choose the bulk diffusivity in (120) to be $D_{R}^{*}$ instead of $D^{*}$. We therefore repeated the calculations for $C_{s}$ with the new estimates of $D_{R}^{*}$ and found that in most cases the Brinkman estimate of $C_{s}$ was still quite close to that obtained via the exact analysis. The reason for this is that $C_{s}$ varies only as a square root of the diffusivity and the maximum deviation between $D^{*}$ and $D_{R}^{*}$ for the cases considered here is less than $30 \%$. Finally, one may also inquire what choices of $D_{R}^{*}$ will yield the estimates of $C_{s}$ obtained via the approximate theory of Chang and Acrivos to coincide with $C_{s}$ obtained from the exact analysis. We found that the estimates of $D_{R}^{*}$ thus obtained differed significantly from $D^{*}$ even for very slow reactions. Since this is unphysical, we conclude that the approximate theory of
Chang and Acrivos does not yield reliable estimates of $C_{s}$ or $S$ for the problem considered in this section.

\section{B. Asymptotic analysis for slow reactions and small volume fractions}

In this section we consider the case of small $\chi$ and $\mathscr{R}$. As in the case of shear flow, we anticipate that the concentration field can be adequately approximated by considering only a few terms in the formal solution. Accordingly, we consider only the terms containing $A_{00}$ and $A_{10}$, which correspond, respectively, to the concentration monopole and dipole. These two constants can be determined by considering $Y_{0}^{0}$ and $Y_{1}^{o}$ terms in the expansion of $c$ around each sphere. Thus writing down explicitly these terms we have

$$
\begin{aligned}
& E_{00}^{m}=a L_{0} D_{00}^{m}, \\
& E_{10}^{m}=a^{3} L_{1} D_{10}^{m},
\end{aligned}
$$

where

$$
\begin{aligned}
E_{00}^{m}= & 2 A_{00}^{m} \text { and } E_{10}^{m}=-2 A_{10}^{m}, \\
D_{00}^{m}= & \frac{8 \pi}{\tau} \sum_{m<\alpha}(m-\alpha) l A_{00}^{\alpha}+A_{10}^{\alpha} \\
& +\sum_{\alpha} A_{00}^{\alpha} \Psi_{1, m \alpha}^{*}+A_{10}^{\alpha} \frac{\partial \Psi_{1, m \alpha}^{*}}{\partial x_{1}}, \\
D_{10}^{m}= & \frac{8 \pi}{\tau} \sum_{m<\alpha} A_{00}^{\alpha}+O\left(A_{10}^{\alpha}\right) .
\end{aligned}
$$

Here $\Psi_{1, m \alpha}^{*}$ is $\Psi_{1}$ evaluated at the vector difference $r^{m}-\mathbf{r}^{\alpha}$ with the singular part omitted for $m=\alpha$. Similar convention applies to the derivative of $\Psi_{1}$ with respect to $x_{1}$ in (125). Now we expect the concentration to decay exponentially with $x_{1}$ and hence seek the solution of the above equations in the form

$$
A_{00}^{\alpha}=A e^{-\alpha \sigma(\chi)} \text { and } A_{10}^{\alpha}=B e^{-\alpha \sigma(\chi)},
$$

where, by comparison with (121), we expect that $\sigma=O\left(\chi^{3}\right)$. Now substitution of (127) into (123)-(126) yields

$$
B=\frac{\beta-1}{\beta+2} \frac{4 \pi a^{3}}{\tau} \frac{e^{-\sigma}}{1-e^{-\sigma}} A,
$$

where use has been made of the asymptote $L_{1}=(1-\beta) /$ $(2+\beta)+O\left(z^{2}\right)$ [cf. (116) for the definition of $z$ ]. Now, using the relations

$$
\begin{aligned}
\sum_{\alpha} A_{00}^{\alpha} \Psi_{1, m \alpha}^{*} & \\
= & A_{00}^{m} \sum_{\alpha} \Psi_{1, m \alpha}^{*}+\frac{A^{m} \sigma^{2}}{2} \sum_{\alpha}(m-\alpha)^{2} \Psi_{1, m \alpha}^{*}+\cdots, \\
= & c_{1} A^{m}+O\left(\sigma^{2}\right), \quad c_{1}=\sum_{\alpha} \Psi_{1, m \alpha}^{*}, \\
\sum_{\alpha} A_{10}^{\alpha} \frac{\partial \Psi_{1, m \alpha}^{*}}{\partial x_{1}} & =A_{10}^{m} \sum_{\alpha} \sigma(\alpha-m) \frac{\partial \Psi_{1, m \alpha}}{\partial x_{1}}+\cdots \\
& =O\left(\sigma A_{10}^{m}\right),
\end{aligned}
$$

and 


$$
\sum_{\alpha>m}(m-\alpha) A_{00}^{\alpha}=-\frac{A_{00}^{m} e^{-\sigma}}{\left(1-e^{-\sigma}\right)^{2}},
$$

we obtain

$$
\begin{aligned}
D_{00}^{m}= & \frac{8 \pi A_{00}^{m}}{\tau} \frac{e^{-\sigma}}{\left(1-e^{-\sigma}\right)^{2}} \\
& \times\left(-l+\frac{\beta-1}{\beta+2} \frac{4 \pi a^{3}}{\tau} e^{-\sigma}\right)+A_{00}^{m} c_{1} .
\end{aligned}
$$

Now, combining (132) with (122) and using the asymptotic expansion of $L_{0}$,

$$
\begin{aligned}
L_{0}= & -\frac{\chi^{2} \mathscr{R}}{3}+\frac{\chi^{4} \mathscr{R}^{2}}{3 \beta}\left(\frac{\beta}{3}+\frac{1}{15}\right) \\
& -\frac{\chi^{6} \mathscr{R}^{3}}{3 \beta^{2}}\left[\left(\frac{\beta}{3}+\frac{1}{15}\right)^{2}+\frac{1}{525}\right]+O\left(\frac{\chi^{8} \mathscr{R}^{4}}{\beta^{4}}\right),
\end{aligned}
$$

we obtain

$$
2 \sigma^{2}=L_{0}\left(-\frac{8 \pi l}{\tau}+c_{1}^{\prime} \sigma^{2}+\frac{32 \pi^{2}}{\tau^{2}} \frac{\beta-1}{\beta+2} a^{3}+O\left(a^{6}\right)\right),
$$

where $c_{1}^{\prime}=c_{1}+2 \pi l / 3 \tau$. Now $\sigma$ can be determined by successive approximations. The leading term for $\sigma$ is

$$
\sigma^{2}=4 \pi l a^{3} \mathscr{R} / 3 h^{2} \tau=\phi l^{2} \mathscr{R} / h^{2},
$$

in agreement with the Brinkman approximation [cf. (121)]. The above relation can be solved further to determine $\sigma^{2}$ to $O\left(a^{7}\right)$, and by defining the bulk diffusivity via the Brinkman approximation, i.e., $D_{R}^{*} \equiv \phi \mathscr{R} l^{2} / h^{2} \sigma^{2}$, we obtain the following expression for $D_{R}^{*}$ :

$$
\begin{aligned}
\frac{1}{D_{R}^{*}}= & -\frac{1}{3}\left(1+\frac{1}{5 \beta}\right) \mathscr{R} \chi^{2} \\
& -\left(\frac{c_{1}^{\prime} h \mathscr{R}}{6}+\frac{\beta-1}{\beta+2} \frac{4 \pi h^{3}}{\tau l}\right) \chi^{3} \\
& +\left[\left(\frac{1}{3}+\frac{1}{15 \beta}\right)^{2}+\frac{1}{525 \beta^{2}}\right] \mathscr{R}^{2} \chi^{4}+O\left(\chi^{9 / 2}\right) .
\end{aligned}
$$

We note that if we take $\mathscr{R}=0$ we recover the well-known Maxwell result $D_{R}^{*}=D^{*}=1+3 \phi(\beta-1) /(\beta+2)$ valid for the dilute arrays of spheres. The constant $c_{1}^{\prime} h$ equals, respectively, $-2.2838,-3.1790$, and -3.2422 for the simple, body-centered, and face-centered cubic arrays. Substituting its value in (136) yields the following expression for the case of $\beta=\infty$ and the body-centered cubic array:

$$
\begin{aligned}
\frac{1}{D_{R}^{*}}=1 & -\frac{\mathscr{R} \chi^{2}}{3}+(0.530 \mathscr{R}-2.041) \chi^{3} \\
& +\frac{\mathscr{R}^{2} \chi^{4}}{9}+O\left(\chi^{9 / 2}\right) .
\end{aligned}
$$

Thus we see that the effective diffusivity in the presence of a chemical reaction is greater than that of a chemically passive medium, at least to the leading order in $\mathscr{R}$. The above expression was compared with the estimate of $D_{R}^{*}$ obtained from the numerical calculations and the agreement between the two was found to be excellent for $\chi=0.3$ for $\mathscr{R}<0.4$. Finally, the case of $\beta=\infty$ can also be regarded as a case of surface reaction as mentioned earlier. Using $L_{n}$ given by (117), an independent analysis similar to the one outlined above yielded

$$
\begin{aligned}
1 / D_{R}^{*}= & 1-\mathscr{R}_{s} \chi+\left(1-1.589 \mathscr{R}_{s}^{-1}\right) \mathscr{R}_{s}^{2} \chi^{2} \\
& -\left(1+3.179 \mathscr{R}_{s}^{-1}+2.042 \mathscr{R}_{s}^{-3}\right) \mathscr{R}_{s}^{3} \chi^{3}+\cdots
\end{aligned}
$$

We notice that not all of the terms in the above expression can be derived directly from (137) by substituting $\mathscr{R}=3 \mathscr{R}_{s} / \chi$. The reason for this apparent discrepancy lies in the fact that the higher-order terms in (137) which are multiplied by powers of $\mathscr{R}$ can give lower-order terms in the expansion of $\chi$ upon the substitution of $3 \mathscr{R}_{s} / \chi$ for $\mathscr{R}$.

\section{CONCLUDING REMARKS}

In this paper we have presented the planar periodic singular solutions of the Stokes and Laplace equations and their applications to various transport problems near the "interface" of a porous medium with a continuous-phase medium. The transport processes considered here were (i) a shear flow past a porous surface; (ii) a flow in the entrance region of a porous medium; (iii) a heat conduction in the entrance region; and (iv) a diffusion accompanied by a chemical reaction. The results of the exact analysis of each of the above processes were compared with the predictions of two approximate continuum theories referred to as the Brinkman and Chang-Acrivos approximations. It was found that in case (i) the Brinkman approximation gave reasonably good estimates of the velocity slip coefficient and the velocity decay rates up to $\phi=0.15$ for all three cubic arrays and that the Chang-Acrivos approximation gave excellent estimates for nearly the complete range of $\phi$. The agreement for the pressure and temperature slip coefficients in the case of problems (ii) and (iii) with the approximate theories, however, was not satisfactory in that the approximate theories predicted zero values for these coefficients whereas the exact analysis yielded small nonzero values. Since in the case of a periodic array, the particle distribution function near the "interface" is essentially the same as in the bulk, the approximate theories estimate the entrance length related effective properties to be the same as in the bulk and thereby give the estimates of the entrance lengths to be zero. In view of the fact that the exact analysis showed that the entrance lengths in problems (ii) and (iii) are less than approximately one unit cell width, we must regard the approximation made in the continuum theories as quite reasonable. Finally, since the estimates of the bulk diffusivities of reacting media were not available we restricted ourselves to the comparison of the exact numerical results with the approximate theories for slow to moderately fast reactions where the bulk diffusivities are not too different from the diffusivities of nonreacting media. We found in this case that although both approximate theories gave reasonably good estimates of the concentration slips for a fairly wide range of $\chi$, the approximate theory of Brinkman gave more accurate estimates than the theory of Chang and Acrivos. Brinkman theory was also employed to obtain the estimates of the bulk diffusivities of reacting media. 
In addition to the problems studied here, the planar singular solutions can also be employed in the study of a number of other problems. Essentially all the problems concerned with the determination of the bulk properties that required the triply periodic singular solutions developed by Hasimoto $^{21}$ can also be solved using the planar singular solutions. The latter gives more flexibility in studying the arrays that do not have cubic symmetry and may, in particular, be more useful in studying the transport processes in random suspensions. We have recently found a formal solution for the velocity field for $N$ randomly arranged spheres per unit planar cell and we are currently using this solution to obtain estimates of various transport properties of disordered systems.

\section{ACKNOWLEDGMENTS}

This paper was written while one of us (A.S.) was spending the summer at The Johns Hopkins University.

This work was partially supported by the National Science Foundation under Grant No. CBT-8800451.

'G. Bossis and J. F. Brady, J. Chem. Phys. 80, 5141 (1984).

${ }^{2}$ J. F. Brady and G. Bossis, J. Fluid Mech. 155, 105 (1985).

${ }^{3}$ P. Mazur and W. van Saarloos, Physica A 115, 21 (1982).
${ }^{4}$ C. W. J. Beenakker and P. Mazur, Physica A 120, 388 (1983):

'A. J. C. Ladd, J. Chem. Phys. 88, 5051 (1988).

'A. S. Sangani and C. Yao, J. Appl. Phys. 63, 1334 (1988).

${ }^{7}$ A. S. Sangani and C. Yao, Phys. Fluids 31, 2426 (1988).

${ }^{8}$ A. S. Sangani and C. Yao, Phys. Fluids 31, 2435 (1988).

${ }^{9}$ A. Acrivos and E. Chang, Phys. Fluids 29, 3 (1986).

${ }^{10}$ E. Y. Chang, B. S. Yendler, and A. Acrivos, in Advances in Multiphase Flow and Related Problems, edited by G. Papanicolaou (SIAM, Philadelphia, 1986).

"'E. Chang and A. Acrivos, J. Appl. Phys. 59, 375 (1986).

${ }^{12}$ E. Y. Chang and A. Acrivos, Chem. Eng. Commun. 58, 165 (1987).

${ }^{13}$ E. Y. Chang and A. Acrivos, J. Appl. Phys. 62, 771 (1987).

${ }^{14}$ R. E. Larson and J. J. L. Higdon, J. Fluid Mech. 166, 449 (1986).

${ }^{15}$ R. E. Larson and J. J. L. Higdon, J. Fluid Mech. 178, 119 (1987).

${ }^{16}$ K. Ishii, J. Phys. Soc. Jpn. 46, 675 (1979).

${ }^{17}$ H. C. Brinkman, Appl. Sci. Res. Sect. A 1, 27 (1947).

${ }^{1 k}$ M. Born and R. D. Misra, Proc. Cambridge Philos. Soc. 36, 446 (1940).

${ }^{19} \mathrm{E}$. W. Hobson, The Theory of Spherical and Ellipsoidal Harmonics (Cambridge U.P., Cambridge, 1931).

${ }^{20}$ P. G. Saffman, Stud. Appl. Math. 50, 93 (1971).

${ }^{21}$ H. Hasimoto, J. Fluid Mech. 5, 317 (1959).

${ }^{22}$ A. A. Zick and G. M. Homsy, J. Fluid Mech. 115, 13 (1982).

${ }^{23}$ A. S. Sangani and A. Acrivos, Int. J. Multiphase Flow 8, 343 (1982).

${ }^{24}$ S. Kim and W. B. Russel, J. Fluid Mech. 154, 269 (1985).

${ }^{25}$ J. Koplik, H. Levine, and A. Zee, Phys. Fluids 26, 2864 (1984).

${ }^{26}$ Y. A. Buyevich and I. N. Shchelchkova, Prog. Aerospace Sci. 18, 121 (1978).

${ }^{27}$ A. S. Sangani, J. Appl. Math. Phys. (ZAMP) 38, 542 (1987).

${ }^{28}$ B. U. Felderhof, Physica A 130, 34 (1985).

${ }^{29}$ K. Mattern and B. U. Felderhof, Physica A 143, 21 (1987). 